Pacific Journal of Mathematics

ACTIONS OF DISCRETE AMENABLE GROUPS ON INJECTIVE 
Pacific Journal of Mathematics

ACTIONS OF DISCRETE AMENABLE GROUPS ON INJECTIVE 


\title{
ACTIONS OF DISCRETE AMENABLE GROUPS ON INJECTIVE FACTORS OF TYPE $\mathrm{III}_{\lambda}, \lambda \neq 1$
}

\section{Colin E. Sutherland and Masamichi Takesaki Dedicated to the memory of Professor Henry Abel Dye}

\begin{abstract}
Actions $\alpha$ of a discrete amenable group $G$ on an injective factor $\mathscr{M}$ of type III $_{\lambda}, \lambda \neq 1$, are classified up to cocycle conjugacy in terms of the module $\bmod (\alpha)$, the characteristic invariant $\chi_{\alpha}$ and the modular invariant $\nu_{\alpha}$ of $\alpha$. These invariants live on the flow of weights. It is also shown that each element of $H^{1}(\mathscr{F}(\mathscr{M}))$, the first cohomology group of the flow of weights, has a $C^{\infty}$-representative cocycle.
\end{abstract}

0. Introduction. The purpose of this paper is to provide a classification, up to cocycle conjugacy, of the possible actions of a discrete amenable group $G$ on an injective factor of type $\mathrm{III}_{\lambda}, \lambda \neq 1$; it thus represents a continuation of the line of research initiated in $[1,2]$ and continued in $[7, \mathbf{1 1}, \mathbf{8}, \mathbf{1 5}]$. We do not touch the remaining classification problem (that of actions on the injective factor of type $\mathrm{III}_{1}$ ) here. This problem requires a deeper understanding of actions of $\mathbf{R}$ on the injective factors of type $\mathrm{II}_{1}$ and type $\mathrm{II}_{\infty}$.

The principal tools involved in the classification are drawn from the crossed product structure theorem for factors of type III of [16], from ideas involved in the flow of weights [4], from the authors' previous work [15] on classification of actions of discrete amenable groupoids on semifinite injective von Neumann algebras, and from techniques of reduction from continuous to discrete groupoids related to [5]. We will draw heavily on results from these sources.

To help orient the reader, we first consider an action $\alpha$ of a discrete amenable group $G$ on an injective factor $\mathscr{M}$ of type $\operatorname{III}_{\lambda}, 0<\lambda<1$. In this case, one may use the discrete decomposition $\mathscr{M}=\mathscr{N} \rtimes \mathbf{Z}$ of $\mathscr{M}$ (see e.g. [3]) to reduce the problem to one of classification of an action of $G \times \mathbf{Z}$ on the $\mathrm{II}_{\infty}$ injective factor $\mathscr{N}$. Unfortunately, this method does not generalize to the type $\mathrm{III}_{0}$ case, so we will briefly describe an approach which does generalize, based on the continuous decomposition of [16]. We may reduce to the case where $\alpha$ admits an invariant dominant weight $\phi$ with $\sigma_{T}^{\phi}=\mathrm{id}$, where $T=-2 \pi / \log \lambda$. Observe that in this situation, $\left\{\alpha_{g}: g \in G\right\}$ leaves the centralizer $\mathscr{M}_{\phi}$ of 
$\phi$ invariant, and hence gives rise to an action $\gamma_{\alpha}$ of $G$ on the centre $\mathscr{C}_{\phi}$ of $\mathscr{M}_{\phi}$ by restriction. This action commutes with the restriction of the dual flow $\left\{\hat{\sigma}_{t}^{\phi}: t \in \mathbf{R}\right\}$ on $\mathscr{M}_{\phi}$ to $\mathscr{C}_{\phi}$; since this latter flow is periodic of period $-\log \lambda$, we obtain a homomorphism $\gamma_{\alpha}^{0}$ of $G$ into $\mathbf{R} /(-\log \lambda) \mathbf{Z}$. Of course, the restriction of the dual flow to $\mathscr{C}_{\phi}$ is nothing but the (smooth) flow of weights, [4], and the action $\gamma$ is precisely mod $\circ \alpha$, where mod is as defined in [4, p. 459]. Recall that the flow of weights completely determines $\mathscr{M}$. We will also need to look at the relation of $\left\{a_{g}: g \in G\right\}$ to the group of automorphisms of $\mathscr{M}$ of the form Ad $u \circ \sigma_{t}^{\phi}, t \in \mathbf{R}, u$ a unitary in $\mathscr{M}$. This group, identified in [3] as the group $\operatorname{Cnt}(\mathscr{M})$ of centrally trivial automorphisms, has the advantage that if $N(\alpha)=\alpha^{-1}(\operatorname{Cnt}(\mathscr{M}))$, then each automorphism $\alpha_{n}, n \in N(\alpha)$, becomes inner on restriction to $\mathscr{M}_{\phi}$ : indeed, if we choose $u(n) \in \mathscr{U}(\mathscr{M})$, $t_{n} \in \mathbf{R}$ with $\alpha_{n}=\operatorname{Ad} u(n) \circ \sigma_{t_{n}}^{\phi}$ for $n \in N(\alpha), u(n) \in \mathscr{M}_{\phi}$. Also it is routine to check that there are functions $\mu: N \times N \rightarrow \mathbf{T}, \lambda: N \times G \rightarrow \mathbf{T}$ such that for $m, n \in N, g \in G$,

$$
u(m) u(n)=\mu(m, n) u(m n)
$$

and

$$
\alpha_{g}\left(u\left(g^{-1} n g\right)\right)=\lambda(n, g) u(n) .
$$

As in $[7,11,15]$, the pair $(\mu, \lambda)$ gives rise to an element $\chi_{\alpha}=[\lambda, \mu]$ in the group $\Lambda\left(G, N(\alpha)\right.$, T). Finally, although $n \rightarrow t_{n} \in \mathbf{R}$ is not necessarily a homomorphism, it is modulo $T \mathbf{Z}$, so we can obtain a homomorphism $\nu_{\alpha}$ of $N$ into $\mathbf{R} / T \mathbf{Z}$. We may thus construct from $\alpha$ an element $\left(\gamma_{\alpha}^{0}, \chi_{\alpha}, \nu_{\alpha}\right) \in \operatorname{Hom}(G, \mathbf{R} / \mathbf{Z} \log \lambda) \times \Lambda(G, N(\alpha), \mathbf{T}) \times \operatorname{Hom}(N, \mathbf{R} / T \mathbf{Z})$.

Note that it is not completely straight-forward to check that this element is a cocycle conjugacy invariant for $\alpha$, since the whole construction has been predicated on the choice of an invariant, dominant, periodic weight $\phi$. To circumvent this problem we need to use more of the machinery from [4]; at the same time this will give an invariant adequate to classify actions on injective factors of type III $_{0}$ as well. So let $\mathscr{M}$ be an injective factor of type $\operatorname{III}_{\lambda}, \lambda \neq 1$, and let $\operatorname{Cnt}(\mathscr{M})$ denote the group of centrally trivial automorphisms of $\mathscr{M}$. One knows from $[3,4]$, that if $\phi$ is any dominant weight on $\mathscr{M}$ every element of $\operatorname{Cnt}(\mathscr{M})$ is of the form $\operatorname{Ad} u \circ \bar{\sigma}_{c}^{\phi}$ where $u \in \mathscr{M}$ is unitary, $c$ is a cocycle on the flow of weights $\mathscr{F}(\mathscr{M})$ of $\mathscr{M}$, and $\bar{\sigma}$ is the extended modular automorphism group of $\mathscr{M}$. Thus for each $n \in N(\alpha)=\alpha^{-1}(\operatorname{Cnt}(\mathscr{M}))$ we may choose $u(n) \in \mathscr{U}(\mathscr{M})$ and $c(n) \in Z^{1}(\mathscr{F}(\mathscr{M}))$ such that

$$
\alpha_{n}=\operatorname{Ad} u(n) \circ \bar{\sigma}_{c(n)}^{\phi}, \quad n \in N(\alpha) .
$$


The pair $(u(n), c(n))$ gives rise to two related invariants; one is a "characteristic invariant" $\chi_{\alpha}$ in the sense of [7], which comes from comparing the expressions for $\alpha_{m n}$ and $\alpha_{m} \circ \alpha_{n}$, and for $\alpha_{g^{-1} n g}$ and $\alpha_{g}^{-1} \circ \alpha_{n} \circ \alpha_{g}$. Note that this time there is much greater ambiguity in the expression for $\alpha_{n}, n \in N(\alpha)$, since $\bar{\sigma}_{c}^{\phi}$ is inner precisely when the cocycle $c$ is a coboundary i.e. $c \in B^{1}(\mathscr{F}(\mathscr{M}))$. The corresponding characteristic invariant thus falls in $\Lambda(G, N(\alpha), \mathscr{U}(\mathscr{F}(\mathscr{M})))$. The other invariant, here called the "modular invariant" is the homomorphism $\nu_{\alpha}: N \rightarrow H^{1}(\mathscr{F}(\mathscr{M}))$ given by $\nu_{\alpha}(n)=\left[c_{n}\right]$. Finally, we have the homomorphism $\gamma_{\alpha}: G \rightarrow \operatorname{Aut}(\mathscr{F}(\mathscr{M}))$, the automorphisms commuting with the flow of weights, given by $\gamma_{\alpha}=\bmod \circ \alpha$. This time, it is clear from the functorial nature of the flow of weights that the orbit of $\left(\gamma_{\alpha}, \chi_{\alpha}, \nu_{\alpha}\right)$ in

$\operatorname{Hom}(G, \operatorname{Aut}(\mathscr{F}(\mathscr{M}))) \times \Lambda(G, N(\alpha), \mathscr{U}(\mathscr{F}(\mathscr{M}))) \times \operatorname{Hom}\left(N, H^{1}(\mathscr{F}(\mathscr{M}))\right)$ under $\operatorname{Aut}(\mathscr{M})$ is a cocycle conjugacy invariant for $\alpha$.

Our main theorem is a converse to this: if $\alpha$ and $\beta$ are actions of a discrete amenable group $G$ on an injective factor $\mathscr{M}$ of type $\mathrm{III}_{\lambda}, \lambda \neq 1$, then $\alpha$ and $\beta$ are cocycle conjugate if and only if, up to an automorphism of $\mathscr{F}(\mathscr{M}), N(\alpha)=N(\beta)$, say $N$, and $\left(\gamma_{\alpha}, \chi_{\alpha}, \nu_{\alpha}\right)=\left(\gamma_{\beta}, \chi_{\beta}, \nu_{\beta}\right)$. We also give, in Theorem 5.14, precise conditions under which a system $(\gamma, \chi, \nu) \in \operatorname{Aut}(\mathscr{F}(\mathscr{M})) \times \Lambda(G, N, \mathscr{U}(\mathscr{F}(\mathscr{M}))) \times \operatorname{Hom}\left(N, H^{1}(\mathscr{F}(\mathscr{M}))\right)$ is the system of invariants for an action $\alpha$ of $G$ on $\mathscr{M}-\gamma$ can be arbitrary but $\chi$ is restricted and the modular invariant $\nu$ must be linked to $\chi$ in a suitable manner.

The method of proof is to show that up to cocycle conjugacy, $\alpha$ and $\beta$ admit invariant dominant weights, $\phi$ and $\psi$ say, and that in the associated decompositions $\mathscr{M}=\mathscr{M}_{\phi} \rtimes \mathbf{R}=\mathscr{M}_{\psi} \rtimes \mathbf{R}, \alpha$ and $\beta$ fix the one parameter unitary groups corresponding to R-actions on $\mathscr{M}_{\phi}$ and $\mathscr{M}_{\psi}$. We may thus consider actions $\alpha^{\prime}$ and $\beta^{\prime}$ of $G \times \mathbf{R}$ on $\mathscr{M}_{\phi}$ and $\mathscr{M}_{\psi}$, and show that their characteristic invariants are determined completely by $\left(\gamma_{\alpha}, \chi_{\alpha}, \nu_{\alpha}\right)$. Note that $\mathscr{M}_{\phi}$ and $\mathscr{M}_{\psi}$ are of the form $\mathscr{A} \bar{\otimes} \mathscr{R}_{0,1}$, where $\mathscr{R}_{0,1}$ is the injective factor of type $\mathrm{II}_{\infty}$ and $\mathscr{A}$ is abelian; an extension of the results of [15] now guarantees cocycle conjugacy of the actions $\alpha^{\prime}$ and $\beta^{\prime}$, and a little further work secures cocycle conjugacy of $\alpha$ and $\beta$.

The plan of the paper is as follows. In $\S 1$ we collect several preliminary results; $\S 1.1$ discusses partial crossed products in relation to cocycle conjugacy; $\S 1.2$ provides a "point-realization" of actions of separable locally compact groups on algebras of the form $\mathscr{A} \bar{\otimes} \mathscr{P}$, 
(where $\mathscr{A}$ is an abelian von Neumann algebra and $\mathscr{P}$ a factor), via actions of a suitable groupoid on $\mathscr{P} ; \S 1.3$ proves an isomorphism of the relative cohomology group $\Lambda(G, N, \mathscr{U}(\mathscr{A}))$ with the relative cohomology groups $\Lambda(\mathscr{G}, \mathscr{N}, \mathbf{T})$ in the situation when $G$ is a separable locally compact group, $N$ is normal, and $\mathscr{G}, \mathscr{N}$ are discrete groupoids arising as reductions to a complete lacunary section of the groupoids obtained from point realizations of the actions of $G$ and $N$ on $\mathscr{A}$.

In $\S 2$, we determine which characteristic invariants

$$
\chi \in \Lambda(G, N, \mathscr{U}(\mathscr{A}))
$$

extend to elements of $\Lambda(G \times \mathbf{R}, N, \mathscr{U}(\mathscr{A}))$ and parametrize the extensions for discrete $G$-this is vital for us to be able to describe which extended characteristic invariants arise from actions. This is done by exhibiting three exact sequences involving $\Lambda(G \times \mathbf{R}, N, \mathscr{U}(\mathscr{A}))$.

Section 3 proves that any cohomology class of $H^{1}(\mathbf{R}, \mathscr{U}(\mathscr{A}))$ has a $C^{\infty}$-representative.

In $\S 4$, we give a classification of actions $\alpha$ of $G \times \mathbf{R}$ on $\mathscr{N}=\mathscr{A} \bar{\otimes} \mathscr{R}_{0,1}$ under the assumption that $\mathscr{N}$ carries a trace $\operatorname{Tr}$ such that $\operatorname{Tr} \circ \alpha_{g}=\operatorname{Tr}$, $g \in G$, and $\operatorname{Tr} \circ \alpha_{s}=e^{-s} \operatorname{Tr}, s \in \mathbf{R}$, and that $\mathbf{R}$ acts ergodically on $\mathscr{A} \neq \mathrm{C}$. The proof depends heavily on the reduction techniques of $\S \S 1.2$ and 1.3, and the authors' previous results [15].

Finally, we prove in $\S 5$ our main theorem, Theorem 5.9 by making use of all the preliminary material and our result of $\S 4$.

1. Preliminaries. Here we collect several preliminary results which will be needed in the sequel, and which may have independent interest. Some of them are almost folklore, but we include proofs for completeness sake. All von Neumann algebras occurring herein are assumed to be separable in the sense of having separable predual.

1.1. Partial crossed products. Let $\mathscr{M}$ be a von Neumann algebra acting on a Hilbert space $\mathscr{H}$, and let $G=N \rtimes_{\gamma} H$ be a semi-direct product locally compact group with $N$ normal in $G$ and $H$ acting on $N$ via $\gamma: H \rightarrow \operatorname{Aut}(N)$. Thus

$$
\begin{gathered}
G=\{(n, h): n \in N \quad \text { and } h \in H\}, \\
(n, h)(m, k)=\left(n \gamma_{h}(m), h k\right) ;
\end{gathered}
$$

we identify $N$ and $H$ with the subgroups $N \times\{e\}$ and $\{e\} \times H$ of $G$ in the usual way.

Note that if $\alpha: G \rightarrow \operatorname{Aut}(\mathscr{M})$ is a (strong* continuous) action of $G$ on $\mathscr{M}$, there is a natural action $\bar{\alpha}$ of $H$ on $\mathscr{M} \rtimes_{\alpha} N$. In fact, if $\pi_{\alpha}(x)$, 
$x \in \mathscr{M}$, and $\lambda^{\alpha}(n), n \in N$, are the usual generators of $\mathscr{M} \rtimes_{\alpha} N$ on $L^{2}(N ; \mathscr{H})$ given by:

$$
\begin{aligned}
\left(\pi^{\alpha}(x) \xi\right)(n) & =\alpha_{n}^{-1}(x) \xi(n), & x \in \mathscr{M} ; \\
\left(\lambda^{\alpha}(m) \xi\right)(n) & =\xi\left(m^{-1} n\right), & m, n \in N
\end{aligned}
$$

for $\xi \in L^{2}(N, \mathscr{H})$, we have for each $h \in H$,

$$
\begin{array}{ll}
\bar{\alpha}_{h}\left(\pi^{\alpha}(x)\right) & =\pi^{\alpha}\left(\alpha_{h}(x)\right), \quad x \in \mathscr{M} ; \\
\bar{\alpha}_{h}\left(\lambda^{\alpha}(n)\right) & =\lambda^{\alpha}\left(\gamma_{h}(n)\right)=\lambda^{\alpha}\left(h n h^{-1}\right), \quad n \in N .
\end{array}
$$

Proposition 1.1. Suppose that $\alpha$ and $\beta$ are actions of $G=N \rtimes_{\gamma} H$ on $\mathscr{M}$, and $\bar{\alpha}, \bar{\beta}$ are respectively the actions of $H$ on $\mathscr{M} \rtimes_{\alpha} N$ and $\mathscr{M} \rtimes_{\beta} N$ defined above. Then if $\alpha$ and $\beta$ are cocycle conjugate as actions of $G$ on $\mathscr{M}$, the actions $\bar{\alpha}$ and $\bar{\beta}$ of $H$ on $\mathscr{M} \rtimes_{\alpha} N$ and $\mathscr{M} \rtimes_{\beta} N$ are also cocycle conjugate.

Proof. By our hypothesis, there is an automorphism $\kappa \in \operatorname{Aut}(\mathscr{M})$ and an element $u \in Z_{\beta}^{1}(G, \mathscr{U}(\mathscr{M}))$ such that

$$
\kappa \circ \alpha_{g} \circ \kappa^{-1}=\operatorname{Ad} u(g) \circ \beta_{g}, \quad g \in G .
$$

Since the case that $u(g)=1, g \in G$, is trivial, we may assume $\kappa$ is trivial.

Define a unitary $U$ on $L^{2}(N ; \mathscr{H})$ by

$$
(U \xi)(n)=u\left(n^{-1}\right)^{*} \xi(n), \quad \xi \in L^{2}(N ; \mathscr{H}), \quad n \in N .
$$

Routine calculations show that $\theta=\operatorname{Ad} U$ defines an isomorphism of $\mathscr{M} \rtimes_{\alpha} N$ onto $\mathscr{M} \rtimes_{\beta} N$ with

$$
\begin{aligned}
& \theta\left(\pi^{\alpha}(x)\right)=\pi^{\beta}(x), \quad x \in \mathscr{M} ; \\
& \theta\left(\lambda^{\alpha}(n)\right)=\pi^{\beta}(u(n)) \lambda^{\beta}(n), \quad n \in N .
\end{aligned}
$$

With $v(h)=\pi^{\beta}(u(h)), h \in H$, we have $v \in Z_{\beta}^{1}\left(H, \mathscr{U}\left(\mathscr{M} \rtimes_{\beta} N\right)\right)$. It then follows that $\theta \circ \bar{\alpha}_{h} \circ \theta^{-1}=\operatorname{Ad}(v(h)) \circ \bar{\beta}_{h}, h \in N$.

1.2. Actions on $\mathscr{A} \otimes \mathscr{P}$. In this section, $\mathscr{A}$ denotes an abelian von Neumann algebra, $\mathscr{P}$ a factor and $G$ a separable locally compact group. We are interested in describing the actions $\alpha$ of $G$ on $\mathscr{A} \otimes \mathscr{P}$ by means of the actions of an appropriate measured groupoid $\mathscr{G}$ on $\mathscr{P}$. The case where $G$ is discrete is discussed in [15]; the non-discrete case is also more or less known, but to establish notations and conventions, we 
include the following:

Proposition 1.2. Let $G$ and $\mathscr{A} \bar{\otimes} \mathscr{P}$ be as above, and let $\alpha: G \rightarrow$ $\operatorname{Aut}(\mathscr{A} \bar{\otimes} \mathscr{P})$ be an action. Then there exists a separable compact $G$ space $X$ carrying a quasi-invariant measure $m$, a Borel homomorphism $\alpha: \mathscr{G}=G \ltimes X \rightarrow \operatorname{Aut}(\mathscr{P})$, and an isomorphism $\kappa: \mathscr{A} \bar{\otimes} \mathscr{P} \rightarrow$ $\int_{X}^{\oplus} \mathscr{P} d m(x)$ such that for any $T \in \mathscr{A} \bar{\otimes} \mathscr{P}$ and $g \in G$,

$$
\kappa\left(\alpha_{g}(T)\right)=\int_{X}^{\oplus} \alpha_{\left(g, g^{-1} x\right)}\left(\kappa(T)\left(g^{-1} x\right)\right) d m(x) .
$$

Proof. Let $A \subseteq \mathscr{A} \bar{\otimes} \mathscr{P}$ be a $\sigma$-weakly dense unital separable $C^{*}$ subalgebra such that (i) $\alpha_{g}(A)=A, g \in G$; (ii) the action $\alpha$ of $G$ on $A$ is norm continuous; (iii) $A \cap(\mathscr{A} \otimes \mathbf{C})$ is $\sigma$-weakly dense in $\mathscr{A} \bar{\otimes} \mathbf{C}$. We let $X$ be the spectrum of $A \cap(\mathscr{A} \otimes \mathbf{C})$, and let $G$ act on $X$ via the inverse transpose of the restriction of $\alpha$ to $A \cap(\mathscr{A} \otimes \mathbf{C})$ so that $X$ is a separable compact $G$-space, and carries a quasi invariant measure $m$ with $L^{\infty}(X, m) \cong \mathscr{A}$.

Let $l_{A}$ be the self-representation of $A$, and consider the disintegration:

$$
\iota_{A}=\int_{X}^{\oplus} \pi_{x} d m(x)
$$

of $l_{A}$ with respect to the diagonal algebra $\mathscr{A} \otimes \mathbf{C}$, [17, Theorem 4.8.25]. From the construction of the disintegration, it follows directly that for each $(g, x) \in G \times X$ there is an isomorphism $\beta_{(g, x)}: \pi_{x}(A) \rightarrow \pi_{g x}(A)$ with

$$
\beta_{(g, x)} \circ \pi_{x}(a)=\pi_{g x}\left(\alpha_{g}(a)\right), \quad a \in A .
$$

However, for almost every $x \in X, \pi_{x}(A)^{\prime \prime}$ is isomorphic to $\mathscr{P}$, and for almost every $(g, x) \in G \times X, \beta_{(g, x)}$ extends to an automorphism of $\mathscr{P}$. We thus obtain a Borel map $\beta: G \ltimes X \rightarrow \operatorname{Aut}(\mathscr{P})$ which is a weak (i.e. almost everywhere) homomorphism in the sense of [12]. Since $G \ltimes X$ is locally compact, we may apply [12, Theorem 3.2] to conclude that there is a strict Borel homomorphism $\alpha: G \ltimes X \rightarrow \operatorname{Aut}(\mathscr{P})$ which agrees with $\beta$ almost everywhere. that

If now $\kappa$ is the evident identification of $\mathscr{A} \bar{\otimes} \mathscr{P}$ with $\int_{X}^{\oplus} \mathscr{P} d m(x)$ (so

$$
\kappa(a)=\int_{X}^{\oplus} \theta_{x}\left(\pi_{x}(a)\right) d m(x), \quad a \in A,
$$

where $\theta_{x}: \pi_{x}(A)^{\prime \prime} \rightarrow \mathscr{P}$ is an almost everywhere defined Borel field of isomorphisms), then $\kappa$ and $\alpha: G \ltimes X \rightarrow \operatorname{Aut}(\mathscr{P})$ satisfy the conclusion of the proposition. 
We note in passing that point-realizations as given in the proposition are far from unique; any two point-realizations will agree almost everywhere, up to cohomology, but need not agree on contraction to any saturated conull set of units.

1.3. Reduction of $\Lambda(G, N, \mathscr{U}(\mathscr{A}))$. In this section, $G$ is assumed to be a separable locally compact group with a normal subgroup $N$. Let $\alpha$ be an action of $G$ on an abelian von Neumann algebra $\mathscr{A}$.

We define groups $Z_{\alpha}(G, N, \mathscr{U}(\mathscr{A}))$ and $B_{\alpha}(G, N, \mathscr{U}(\mathscr{A}))$ as follows:

First, $Z_{\alpha}(G, N, \mathscr{U}(\mathscr{A}))$ consists of pairs $(\lambda, \mu)$ such that $\lambda: N \times G \rightarrow$ $\mathscr{U}(\mathscr{A})$ and $\mu: N \times N \rightarrow \mathscr{U}(\mathscr{A})$ are Borel maps satisfying the following conditions:

(i) $\mu \in Z_{\alpha}^{2}(N, \mathscr{U}(\mathscr{A}))$, i.e.

$$
\mu(m, n) \mu(m n, l)=\alpha_{m}(\mu(n, l)) \mu(m, n l), \quad m, n, l \in N \text {; }
$$

(ii) $\alpha_{g}\left(\lambda\left(g^{-1} n g, h\right)\right) \lambda(n, g)=\lambda(n, g h), \quad n \in N, g, h \in G$;

(iii) $\lambda(n, m)=\mu\left(m, m^{-1} n m\right) \mu(n, m)^{*}, \quad n, m \in N$;

(iv) $\lambda(m, g) \alpha_{m}(\lambda(n, g)) \lambda(m n, g)^{*}$

$=\alpha_{g}\left(\mu\left(g^{-1} m g, g^{-1} n g\right) \mu(m, n)^{*}, \quad m, n \in N, g \in G\right.$;

(v) $\mu(m, n)=1$ and $\lambda(m, g)=1$ if any of $m, n \in N$ or $g \in G$ is the identity.

Secondly, $B_{\alpha}(G, N, \mathscr{U}(\mathscr{A}))$ consists of all pairs of the form $\left(\partial_{2} c, \partial_{1} c\right)$ where $c: N \rightarrow \mathscr{U}(\mathscr{A})$ is a Borel map and

$$
\begin{aligned}
&\left(\partial_{1} c\right)(m, n)=c(m) \alpha_{m}(c(n)) c(m n)^{*}, \quad m, n \in N ; \\
&\left(\partial_{2} c\right)(n, g)=\alpha_{g}\left(c\left(g^{-1} n g\right)\right) c(n)^{*}, \quad n \in N, g \in G .
\end{aligned}
$$

Finally $\Lambda_{\alpha}(G, N, \mathscr{U}(\mathscr{A}))$ denotes the quotient group:

$$
\Lambda_{\alpha}(G, N, \mathscr{U}(\mathscr{A}))=Z_{\alpha}(G, N, \mathscr{U}(\mathscr{A})) / B_{\alpha}(G, N, \mathscr{U}(\mathscr{A})) .
$$

Of course, a similar definition holds for any abelian Polish $G$-module in place of $\mathscr{U}(\mathscr{A})$. For details, we refer the reader to [7], [11] and [8] where these groups are defined for finite or discrete $G$ and where an interpretation of $\Lambda_{\alpha}(G, N, \mathscr{U}(\mathscr{A}))$ as well as its elementary properties are discussed.

We now assume that $N$ is discrete and acts trivially on $\mathscr{A}$, that the centralizer $C_{G}(N)$ of $N$ in $G$ acts ergodically on $\mathscr{A}$, and that $G / C_{G}(N)$ is discrete, and hence countable. Let $\left\{T_{g}: g \in G\right\}$ on $\left\{X, m^{\prime}\right\}$ be a point-realization of $\{\mathscr{A}, G, \alpha\}$ as given by Proposition 1.2. By [5, Theorem 2.8], there is a complete lacunary section $B$ for the action $\left\{T_{g}: g \in C_{G}(N)\right\}$. If $\mathscr{G}^{\prime}=G \ltimes_{T} X$ and $\mathscr{N}^{\prime}=N \ltimes_{T} X$ are viewed as measured groupoids with Haar measure and $m^{\prime}$, then by [5, Proposition 3.6] or [12] there is a measure $m$ on $B$ such that $\left\{\left.\mathscr{G}^{\prime}\right|_{B}=\mathscr{G},[m]\right\}$ 
is a reduction of $\left\{\mathscr{G}^{\prime},\left[m^{\prime}\right]\right\}$, and similarly for $\left\{\left.\mathscr{N}^{\prime}\right|_{B}=\mathscr{N},[m]\right\}$. Indeed, the relevant homomorphism $p: \mathscr{G}^{\prime} \rightarrow \mathscr{G}$ may be described as follows: for each $x \in X$, choose $h(x) \in C_{G}(N)$ in such a way that $T_{h(x)}^{-1} x=\pi(x) \in B$ and $x \in X \rightarrow h(x) \in C_{G}(N), x \in X \rightarrow \pi(x) \in B$ are both measurable; we then define $p$ by

$$
p(g, x)=\left(h\left(T_{g} x\right)^{-1} g h(x), \pi(x)\right) .
$$

It then follows that $p$ is the desired homomorphism of $\mathscr{G}^{\prime}$ onto $\mathscr{G}$.

Suppose now that $(\lambda, \mu) \in Z(\mathscr{G}, \mathscr{N}, \mathbf{T})$ as defined in [8, p. 229]. We define an element $(\bar{\lambda}, \bar{\mu})$ of $Z_{\alpha}(G, N, \mathscr{U}(\mathscr{A}))$ as follows: for each $(m, n) \in N \times N, \bar{\mu}(m, n)$ is the element of $\mathscr{A}=L^{\infty}\left(X, m^{\prime}\right)$ represented by the function: $x \in X \rightarrow \mu(p(m, x), p(n, x))$, and for each $(n, g) \in$ $N \times G, \bar{\lambda}(n, g)$ is the element of $\mathscr{A}$ represented by the function: $x \in$ $X \rightarrow \lambda\left(p\left(n, T_{g} x\right), p(g, x)\right)$.

THEOREM 1.3. With notation as above, the map

$$
\bar{p}:(\lambda, \mu) \in Z(\mathscr{G}, \mathcal{N}, \mathbf{T}) \rightarrow(\bar{\lambda}, \bar{\mu}) \in Z_{\alpha}(G, N, \mathscr{U}(\mathscr{A}))
$$

determines a well-defined isomorphism

$$
\tilde{p}: \Lambda(\mathscr{G}, \mathscr{N}, \mathbf{T}) \rightarrow \Lambda_{\alpha}(G, N, \mathscr{U}(\mathscr{A})) .
$$

Proof. The well-definedness of $\bar{p}$ as well as its multiplicativity is obvious.

We first consider surjectivity of $\tilde{p}$. To this end, we prove first that if $(\bar{\lambda}, \bar{\mu}) \in Z_{\alpha}(G, N, \mathscr{U}(\mathscr{A}))$, then we may choose $\mu \in Z^{2}(N, \mathbf{T})$ so that $(\bar{\lambda}, \mu \otimes 1)$ is cohomologous to $(\bar{\lambda}, \bar{\mu})$, where $\mu \otimes 1$ means the constant function: $x \in X \rightarrow \mu(m, n) \in \mathbf{T}$. Let $\mu(m, n, x)$ and $\lambda(m, g, x)$ be functions on $X$ which represent $\bar{\mu}(m, n)$ and $\bar{\lambda}(m, g)$ respectively. Writing $g x$ for $T_{g} x$, and using (1.9), we have $\mu\left(g^{-1} m g, g^{-1} n g, g^{-1} x\right) \mu(m, n, x)^{*}=\lambda(m, g, x) \lambda(n, g, x) \lambda(m n, g, x)^{*}$ for each $m, n \in N, g \in G$ and for almost every $x \in X$. By the lifting theorem of Ionescu-Tulcea [6], we may assume $\mu_{x}(m, n)=\mu(m, n, x)$, $x \in X$, gives an element $\mu_{x} \in Z^{2}(N, \mathbf{T})$ for each $x \in X$. The above identity yields that for each $g \in C_{G}(N)$ and $m, n \in N$,

$$
\mu_{g^{-1} x}(m, n) \mu_{x}(m, n)^{*}=\lambda(m, g, x) \lambda(n, g, x) \lambda(m n, g, x)^{*}
$$

for almost every $x \in X$. By Fubini's theorem, this same identity holds for each $x \in X_{0}$ and almost every $g \in C_{G}(N)$, where $X_{0}$ is a conull subset of $X$. Thus for each $x \in X_{0}$ and $g \in G(x) \subseteq C_{G}(N)$, a conull 
subset of $C_{G}(N)$, we have

$$
\mu_{g^{-1} x} \mu^{*}=\partial_{1} c
$$

where $c(m)=\lambda(m, g, x)$. Hence the map $x \in X \rightarrow\left[\mu_{x}\right] \in H^{2}(N, \mathbf{T})$ is almost everywhere constant on $C_{G}(N)$ orbits. Since $H^{2}(N, \mathbf{T})$ is a separable compact group ( $N$ being discrete and countable), the ergodicity of $C_{G}(N)$ on $\left\{X, m^{\prime}\right\}$ implies that $\left[\mu_{x}\right]$ is constant on $X$. Thus, there exists $\mu \in Z^{2}(N, \mathbf{T})$ such that $[\mu]=\left[\mu_{x}\right]$ for almost every $x \in X$.

So assume $(\bar{\lambda}, \mu \otimes 1) \in Z_{\alpha}(G, N, \mathscr{U}(\mathscr{A}))$. Let $\mathscr{P}=\mathscr{A} \ltimes_{\alpha, \mu} N$ be the twisted crossed product of $\mathscr{A}$ by $N, \alpha$, and the 2-cocycle $\mu$ (see [14]). Since $N$ acts trivially on $\mathscr{A}, \mathscr{P}$ is generated on $L^{2}\left(N, L^{2}\left(X, m^{\prime}\right)\right)=$ $L^{2}(N, \mathscr{H})$ by the operators $a \otimes 1, a \in \mathscr{A}$, and the operators $1 \otimes u^{\mu}(n)$, $n \in N$, where

$$
u^{\mu}(m) \xi(n)=\mu\left(n^{-1}, m\right) \xi\left(m^{-1} n\right), \quad \xi \in l^{2}(N) .
$$

As in [15, p. 1105], $G$ acts on $\mathscr{P}$ via $\beta$, where

$$
\beta_{g}(a \otimes 1)=\alpha_{g}(a) \otimes 1, \quad a \in \mathscr{A},
$$

$$
\beta_{g}\left(1 \otimes u^{\mu}\left(g^{-1} n g\right)\right)=\lambda(n, g) \otimes u^{\mu}(n), \quad g \in G, n \in N .
$$

With $\mathscr{Q}=\left\{u^{\mu}(n): n \in N\right\}^{\prime \prime}$ on $l^{2}(N)$, we have $\mathscr{P} \cong \mathscr{A} \bar{\otimes} \mathscr{Q}$.

Let $(g, x) \in G \ltimes X \rightarrow \gamma_{(g, x)} \in \operatorname{Aut}(\mathscr{Q})$ be a point realization of $\{\mathscr{P}, G, \beta\}$ as provided by Proposition 1.2. A straightforward calculation shows that $\beta_{n}=\operatorname{Ad}\left(u^{\mu}(n)\right), n \in N$, so that after deleting a null set, we may assume $\gamma_{(n, x)}=\operatorname{Ad}\left(u^{\mu}(n)\right)$ on $N \times X$.

Define

$$
\lambda((n, g x),(g, x))=\gamma_{(g, x)}\left(u^{\mu}\left(g^{-1} n g\right)\right) u^{\mu}(n)^{*}
$$

for $((n, g x),(g, x)) \in\left(\mathscr{N}^{\prime} \times \mathscr{G}^{\prime}\right) \cap \mathscr{G}^{\prime(2)}$. Note that the function: $x \in$ $X \rightarrow \lambda((n, g x),(g, x))$ represents $\bar{\lambda}(n, g) \in \mathscr{A}=L^{\infty}\left(X, m^{\prime}\right)$ for each $(n, g) \in N \times G$. Set

$$
c(n, x)=\lambda((n, x),(h(x), \pi(x)))^{-1}, \quad(n, x) \in N \times X .
$$

We claim that

$$
\begin{aligned}
& \mu(m, n)=\mu(m, n) c(m, x) c(n, x) c(m n, x)^{-1} ; \\
& \lambda((n, g x),(g, x)) \\
& \quad=c\left(g^{-1} n g, x\right) \lambda(p(n, g x), p(g, x)) c(n, g x)^{-1}
\end{aligned}
$$

for each $m, n \in N, g \in G$ and almost every $x \in X$. Note that if 
the above identities are true, then $(\bar{\lambda}, \mu \otimes 1)$ is cohomologous, via $c(n, \cdot) \in \mathscr{U}(\mathscr{A})$, to $\bar{p}\left(\left.\lambda\right|_{\mathscr{E}} ;\left.(\mu \otimes 1)\right|_{\mathscr{G}}\right)$, and surjectivity of $\tilde{p}$ follows.

To prove (1.17), we compute as follows:

$$
\begin{aligned}
\lambda((m n, x),(h(x), \pi(x))) & \\
& =\gamma_{(h(x), \pi(x))}\left(u^{\mu}\left(h(x)^{-1} m n h(x)\right)\right) u^{\mu}(m n)^{*} \\
& =\gamma_{(h(x), \pi(x))}\left(u^{\mu}(m n)\right) u^{\mu}(m n)^{*} \quad\left(\text { Note } h(x) \in C_{G}(N)\right) \\
& =\gamma_{(h(x), \pi(x))}\left(u^{\mu}(m) u^{\mu}(n)\right) u^{\mu}(n)^{*} u^{\mu}(m)^{*}, \quad(\text { since } \mu(m, n) \in \mathbf{T}), \\
& =\lambda((m, x),(h(x), \pi(x))) \lambda((n, x),(h(x), \pi(x))),
\end{aligned}
$$

so that $c(m n, x)=c(m, x) c(n, x)$.

To prove (1.18), set

$$
u(n, x)=\gamma_{(h(x), \pi(x))}\left(u^{\mu}(n)\right), \quad n \in N .
$$

We will calculate $\gamma_{(g, x)}\left(u\left(g^{-1} n g, x\right)\right) u(n, g x)^{*}$ in two ways. First, by making use of the definition (1.19), we compute

$$
\begin{aligned}
\gamma_{(g, x)} & \left(u\left(g^{-1} n g, x\right)\right) u(n, g x)^{*} \\
& =\gamma_{(g h(x), \pi(x))}\left(u^{\mu}\left(g^{-1} n g\right)\right) \gamma_{(h(g x), \pi(g x))}\left(u^{\mu}(n)^{*}\right) \\
& =\gamma_{(h(g x), \pi(g x))}\left\{\gamma_{\left(h(g x)^{-1} g h(x), \pi(x)\right)}\left(u^{\mu}\left(g^{-1} n g\right)\right) u^{\mu}(n)^{*}\right\} \\
& =\lambda\left((n, \pi(g x)),\left(h(g x)^{-1} g h(x), \pi(x)\right)\right) \\
& =\lambda(p(n, g x), p(g, x)) .
\end{aligned}
$$

On the other hand, since

$$
u(n, x)=\lambda((n, x),(h(x), \pi(x))) u^{\mu}(n),
$$

we may also calculate

$$
\begin{aligned}
\gamma_{(g, x)} & \left(u\left(g^{-1} n g, x\right)\right) u(n, g x)^{*} \\
= & \gamma_{(g, x)}\left(\lambda\left(\left(g^{-1} n g, x\right),(h(x), \pi(x))\right) u^{\mu}\left(g^{-1} n g\right)\right) u^{\mu}(n)^{*} \\
& \times \lambda((n, g x),(h(g x), \pi(g x)))^{-1} \\
= & c\left(g^{-1} n g, x\right) \lambda((n, g x),(g, x)) c(n, g x)^{-1} .
\end{aligned}
$$

Thus (1.18) follows.

To prove the injectivity of $\tilde{p}$, it suffices to show that if $(\lambda, \mu) \in$ $Z(\mathscr{G}, \mathcal{N}, \mathbf{T})$ and $\tilde{p}(\lambda, \mu)=1$ in $\Lambda_{\alpha}(G, N, \mathscr{U}(\mathscr{A}))$, then $(\lambda, \mu) \in$ $B(\mathscr{G}, \mathcal{N}, \mathbf{T})$. But if $\tilde{p}(\lambda, \mu)=1$, then there is a measurable function $c: N \times X \rightarrow \mathbf{T}$ such that

$$
\begin{aligned}
& \mu(p(m, x), p(n, x))=c(m, x) c(n, x) c(m n, x)^{-1} \\
& \lambda(p(n, g x), p(g, x))=c\left(g^{-1} n g, x\right) c(n, g x)^{-1},
\end{aligned}
$$


where the first equation holds for almost every $x$ and all $m, n \in N$ and the second holds almost everywhere in $x$ for each $g \in G$ and $n \in N$. Let

$$
X_{0}=\left\{x \in X: \lambda(p(n, g x), p(g, x))=c\left(g^{-1} n g, x\right) c(n, g x)^{-1}\right.
$$

for almost every $g \in C_{G}(N)$ and every $\left.n \in N\right\}$.

By Fubini's Theorem, $X_{0}$ is conull in $X$. For each $x \in X_{0}$, let $G(x)$ be the set of $g \in C_{G}(N)$ for which the above identity holds, so that $G(x)$ is conull in $C_{G}(N)$. Also set

$$
G_{0}(x)=\left\{g \in C_{G}(N): p(g, x)=p(e, x)\right\},
$$

and note that $G_{0}(x)$ has a positive measure in $C_{G}(N)$. For $g \in G(x) \cap$ $G_{0}(x)$, and $x \in X_{0}$,

$$
\lambda(p(n, g x), p(g, x))=\lambda(p(n, g x), p(e, x))=1,
$$

so that

$$
c(n, x) c(n, g x)^{-1}=1 .
$$

Thus, we conclude that $c(n, \cdot)$ is constant almost everywhere on the fibres of $\pi$, so there is a measurable function $d$ on $B$ such that $c(n, x)=$ $d(n, \pi(x))$ almost everywhere in $x$. It is now routine to check that $(\lambda, \mu)=\left(\partial_{2} d, \partial_{1} d\right)$ as required.

2. Extension of $\Lambda_{\alpha}(G, N, \mathscr{U}(\mathscr{A}))$ to $\Lambda_{\alpha^{\prime}}(G \times \mathbf{R}, N, \mathscr{U}(\mathscr{A}))$. In this section, $\mathscr{A}$ is an abelian von Neumann algebra, $G$ is a separable locally compact group acting via $\alpha$ on $\mathscr{A}$, which is extended to an action of $G \times \mathbf{R}$ denoted by $\alpha$ again. We will determine which elements of $Z_{\alpha}(G, N, \mathscr{U}(\mathscr{A}))$ can be extended to elements of $Z_{\alpha}(G \times \mathbf{R}, N, \mathscr{U}(\mathscr{A}))$.

LeMMA 2.1. Suppose $(\lambda, \mu) \in Z_{\alpha}(G, N, \mathscr{U}(\mathscr{A}))$ extends to $\left(\lambda^{\prime}, \mu^{\prime}\right) \in$ $Z_{\alpha}(G \times \mathbf{R}, N, \mathscr{U}(\mathscr{A}))$. Then for each $t \in \mathbf{R}$,

$$
\begin{gathered}
\left(\alpha_{t}(\mu) \mu^{*}\right)(m, n)=\lambda^{\prime}(m, t) \alpha_{m}\left(\lambda^{\prime}(n, t)\right) \lambda^{\prime}(m n, t)^{*}, \quad m, n \in N ; \\
\left(\alpha_{t}(\lambda) \lambda^{*}\right)(n, g)=\alpha_{g}\left(\lambda^{\prime}\left(g^{-1} n g, t\right)\right) \lambda^{\prime}(n, t), \quad g \in G .
\end{gathered}
$$

Proof. The first assertion is a consequence of the defining identity (1.9) for $\left(\lambda^{\prime}, \mu^{\prime}\right) \in Z_{\alpha}(G \times \mathbf{R}, N, \mathscr{U}(\mathscr{A}))$.

The second follows since

$$
\begin{aligned}
\left(\alpha_{t}(\lambda) \lambda^{*}\right)(n, g) & =\alpha_{t}\left(\lambda\left(t^{-1} n t, g\right)\right) \lambda^{\prime}(n, g)^{*} \\
& =\lambda^{\prime}(n, g t) \lambda^{\prime}(n, t)^{*} \lambda^{\prime}(n, g)^{*} \\
& =\alpha_{g}\left(\lambda^{\prime}\left(g^{-1} n g, t\right)\right) \lambda^{\prime}(n, g) \lambda^{\prime}(n, t)^{*} \lambda^{\prime}(n, g)^{*} \\
& =\alpha_{g}\left(\lambda^{\prime}\left(g^{-1} n g, t\right)\right) \lambda^{\prime}(n, t)^{*}
\end{aligned}
$$


Thus, any extendable $(\lambda, \mu) \in Z_{\alpha}(G, N, \mathscr{U}(\mathscr{A}))$ is, up to cohomology, invariant under the action of $\mathbf{R}$. Namely, if $\chi \in \Lambda_{\alpha}(G, N, \mathscr{U}(\mathscr{A}))$ is extendable, then $\chi \in \Lambda_{\alpha}(G, N, \mathscr{U}(\mathscr{A}))^{\mathbf{R}}$. The following result characterizes extendable characteristic invariants, and also parametrizes them.

Theorem 2.2. Suppose that $N$ acts trivially on $\mathscr{A}$. Then we have three natural exact sequences which describe the structure of $\Lambda_{\alpha}(G \times \mathbf{R}, N, \mathscr{U}(\mathscr{A}))$ :

(2.3) $\operatorname{Hom}(N, \mathscr{U}(\mathscr{A}))^{G} \rightarrow \operatorname{Hom}\left(N, Z_{\alpha}^{1}(\mathbf{R}, \mathscr{U}(\mathscr{A}))\right)^{G}$

$$
\begin{aligned}
& \rightarrow \Lambda_{\alpha}(G \times \mathbf{R}, N, \mathscr{U}(\mathscr{A})) \rightarrow \Lambda_{\alpha}(G, N, \mathscr{U}(\mathscr{A}))^{\mathbf{R}} \\
& \stackrel{\gamma}{\rightarrow} H_{\alpha}^{1}\left(G, \operatorname{Hom}\left(N, Z_{\alpha}^{1}(\mathbf{R}, \mathscr{U}(\mathscr{A}))\right)\right) .
\end{aligned}
$$

(ii) Under the assumption that $\mathbf{R}$ acts ergodically,

(2.4) $\operatorname{Hom}(N, \mathscr{U}(\mathscr{A}))^{G} \rightarrow \operatorname{Hom}(N, \mathscr{U}(\mathscr{A}) / \mathbf{T})^{G} \rightarrow \Lambda_{\alpha}(G \times \mathbf{R}, N, \mathscr{U}(\mathscr{A}))$

$$
\begin{aligned}
& \rightarrow \Lambda_{\alpha}(G, N, \mathscr{U}(\mathscr{A}))^{\mathbf{R}} \times \operatorname{Hom}\left(N, H_{\alpha}^{1}(\mathbf{R}, \mathscr{U}(\mathscr{A}))\right) \\
& \stackrel{\delta}{\rightarrow} H_{\alpha}^{1}\left(\mathbf{R}, B_{\alpha}(G, N, \mathscr{U}(\mathscr{A}))\right) .
\end{aligned}
$$

(iii) Assuming again that $\mathbf{R}$ acts ergodically, we have

$$
0 \rightarrow \Lambda(G, N, \mathbf{T}) \rightarrow \Lambda_{\alpha}(G \times \mathbf{R}, N, \mathscr{U}(\mathscr{A})) \stackrel{r}{\rightarrow} \operatorname{Hom}\left(N, \dot{H}_{\alpha}^{1}(\mathbf{R}, \mathscr{U}(\mathscr{A}))\right) .
$$

To prove the theorem, we need some preparation.

Suppose $[\lambda, \mu]=\chi \in \Lambda_{\alpha}(G, N, \mathscr{U}(\mathscr{A}))^{\mathbf{R}}$, and choose a Borel map $b:(n, t) \in N \times \mathbf{R} \rightarrow b(n, t) \in \mathscr{U}(\mathscr{A})$ such that

(2.5) $\left(\alpha_{t}(\mu) \mu^{*}\right)(m, n)=b(m, t) b(n, t) b(m n, t)^{*}, \quad m, n \in N$;

(2.6) $\left(\alpha_{t}(\lambda) \lambda^{*}\right)(n, g)=\alpha_{g}\left(b\left(g^{-1} n g, t\right)\right) b(n, t)^{*}, \quad g \in G, t \in \mathbf{R}$.

We then define

$$
c(m, s, t)=b(m, s) \alpha_{s}(b(m, t)) b(m, s+t)^{*}
$$

for $m \in N, s, t \in \mathbf{R}$.

LEMMA 2.3. (i) $c(m, \cdot, \cdot) \in B_{\alpha}^{2}(\mathbf{R}, \mathscr{U}(\mathscr{A}))$;

(ii) $c(\cdot, s, t) \in \operatorname{Hom}(N, \mathscr{U}(\mathscr{A}))$;

(iii) $\alpha_{g}\left(c\left(g^{-1} m g, s, t\right)\right)=c(m, s, t), g \in G$.

In other words, $c$ belongs to $\operatorname{Hom}\left(N, B_{\alpha}^{2}(\mathbf{R}, \mathscr{U}(\mathscr{A}))\right)^{G}$.

Proof. (i) This follows from the definition of $B_{\alpha}^{2}(\mathbf{R}, \mathscr{U}(\mathscr{A}))$ and (2.7). 
(ii) For $m, n \in N$ and $s, t \in \mathbf{R}$, we compute:

$$
\begin{aligned}
b(m, s+t) b(n, s+t) b(m n, s+t)^{*}=\left(\alpha_{s+t}(\mu) \mu^{*}\right)(m, n) \\
\quad=\left(\alpha_{s}(\mu) \mu^{*}\right)(m, n) \alpha_{s}\left(\left(\alpha_{t}(\mu) \mu^{*}\right)(m, n)\right) \\
=b(m, s) b(n, s) b(m n, s)^{*} \alpha_{s}\left(b(m, t) b(n, t) b(m n, t)^{*}\right),
\end{aligned}
$$

so that $c(m n, s, t)=c(m, s, t) c(n, s, t)$.

(iii) This follows from examination of $\alpha_{s+t}(\lambda) \lambda^{*}$ in a manner similar to (ii).

We write $\operatorname{Map}(\mathbf{R}, \mathscr{U}(\mathscr{A}))$ for the group of all Borel maps from $\mathbf{R}$ into $\mathscr{U}(\mathscr{A})$ with pointwise multiplication. Set

$$
\begin{aligned}
& A=\operatorname{Hom}\left(N, Z_{\alpha}^{1}(\mathbf{R}, \mathscr{U}(\mathscr{A}))\right) ; \\
& B=\operatorname{Hom}(N, \operatorname{Map}(\mathbf{R}, \mathscr{U}(\mathscr{A}))) ; \\
& C=\operatorname{Hom}\left(N, B_{\alpha}^{2}(\mathbf{R}, \mathscr{U}(\mathscr{A}))\right),
\end{aligned}
$$

where in the definition of $A, B$ and $C$ we consider only Borel maps in the natural sense. We then have a short exact sequence:

$$
1 \rightarrow A \rightarrow B \stackrel{\partial}{\rightarrow} C \rightarrow 1
$$

where $(\partial b)(n, s, t)=b(n, s) \alpha_{s}(b(n, t)) b(n, s+t)^{*}, s, t \in \mathbf{R}, n \in N$. The surjectivity of $\partial$ may be seen as follows: if $\mathscr{G}$ is the groupoid obtained by point realization of the action of $\mathbf{R}$ on $\mathscr{U}(\mathscr{A}), \mathscr{G}$ is hyperfinite and we have evident identifications

$$
\begin{aligned}
\operatorname{Hom} & \left(N, B_{\alpha^{\prime}}^{2}(\mathbf{R}, \mathscr{U}(\mathscr{A}))\right. \\
\quad= & B_{\alpha^{\prime}}^{2}(\mathbf{R}, \operatorname{Hom}(N, \mathscr{U}(\mathscr{A})))=B^{2}(\mathscr{G}, \hat{N})=\partial(\operatorname{Map}(\mathscr{G}, \hat{N})) \\
\quad= & \partial(\operatorname{Hom}(N, \operatorname{Map}(\mathbf{R}, \mathscr{U}(\mathscr{A})))) .
\end{aligned}
$$

Here $\hat{N}$ is the Pontrjagin dual of $N$, and $\partial$ is the differential in groupoid cohomology. Since $G$ acts on $N$ by conjugation and on $\mathscr{U}(\mathscr{A})$ via $\alpha$, there is an action, denoted again by $\alpha$, of $G$ on the exact sequence (2.8). Thus we obtain a long exact sequence:

$$
1 \rightarrow A^{G} \rightarrow B^{G} \rightarrow C^{G} \stackrel{\phi}{\rightarrow} H_{\alpha}^{1}(G, A) \rightarrow H_{\alpha}^{1}(G, B) \rightarrow \cdots,
$$

where $\phi$ is the connecting map in cohomology [9]. Given

$$
[\lambda, \mu] \in \Lambda_{\alpha}(G, N, \mathscr{U}(\mathscr{A}))^{\mathbf{R}},
$$

we set

$$
\gamma([\lambda, \mu])=\phi(c) \in H_{\alpha}^{1}(G, A),
$$

where $c$ is defined in (2.7). 
LEMMA 2.4. The above map $\gamma$ is well-defined.

Proof. Suppose that $b_{1}$ is another $\mathscr{U}(\mathscr{A})$-valued Borel function on $N \times \mathbf{R}$ satisfying (2.5) and (2.6). Let $c_{1}$ be the element of

$$
\operatorname{Hom}\left(N, B_{\alpha}^{2}(\mathbf{R}, \mathscr{U}(\mathscr{A}))\right)^{G}
$$

associated with $b_{1}$ by (2.7). Then we have $\partial_{1}\left(b b_{1}^{*}\right)=1=\partial_{2}\left(b b_{1}^{*}\right)$, so the map: $(m, s) \in N \times \mathbf{R} \rightarrow b(m, s) b_{1}(m, s)^{*}$ belongs to

$$
\operatorname{Hom}(N, \operatorname{Map}(\mathbf{R}, \mathscr{U}(\mathscr{A})))^{G}=B^{G} .
$$

But

$$
\begin{aligned}
& c(m, s, t) \mathcal{c}_{1}(m, s, t)^{*} \\
& \quad=b(m, s) b_{1}(m, s)^{*} \alpha_{s}\left(b(m, t) b_{1}(m, t)^{*}\right) b(m, s+t) b_{1}(m, s+t)^{*},
\end{aligned}
$$

so that $c c_{1}^{*}=\partial\left(b b_{1}^{*}\right)$. Hence $\phi\left(c c_{1}^{*}\right)=1$.

Now suppose that $(\lambda, \mu)=\left(\partial_{2} a, \partial_{1} a\right)$ for some $a \in \operatorname{Map}(N, \mathscr{U}(\mathscr{A}))$. In this case, we can choose $b$ so that $b(m, t)=\alpha_{t}(a(m)) a(m)^{*}$, and obtain $\phi(c)=1$. This shows that $\gamma$ is well-defined.

Proof of Theorem 2.2. (i) Exactness at $\Lambda_{\alpha}(G, N, \mathscr{U}(\mathscr{A}))^{\mathbf{R}}$ : Suppose $\chi^{\prime}=\left[\lambda^{\prime}, \mu^{\prime}\right] \in \Lambda_{\alpha}(G \times \mathbf{R}, \mathscr{U}(\mathscr{A}))$ and let $\lambda=\left.\lambda^{\prime}\right|_{N \times G}$ and $\mu=\mu^{\prime}$. Then we can take $\lambda^{\prime}(n, t)$ for $b(n, t)$ in (2.5) and (2.6). The identity (1.7) for $\lambda^{\prime}$ implies $c=1$. Thus $\gamma([\lambda, \mu])=1$.

Conversely, suppose $\gamma([\lambda, \mu])=1$. With $b$ and $c$ as in (2.5)-(2.7), we have $\phi(c)=1$, so that we can choose $d \in B^{G}$ such that $c=\partial(d)$, i.e.

$$
\begin{gathered}
c(m, s, t)=d(m, s) \alpha_{s}(d(m, t)) d(m, s+t)^{*} ; \\
\alpha_{g}\left(d\left(g^{-1} m g, t\right)\right)=d(m, t) .
\end{gathered}
$$

Define $\bar{b}(n, t)=b(n, t) d(n, t)^{*}$. By (2.13) and the multiplicativity of $d$ in $m$, we have

$$
\alpha_{t}(\lambda) \lambda^{*}=\partial_{2}(\bar{b}(\cdot, t)) \text { and } \alpha_{t}(\mu) \mu^{*}=\partial_{1}(\bar{b}(\cdot, t)) .
$$

By $(2.11), \bar{b}(n, \cdot) \in Z_{\alpha}^{1}(\mathbf{R}, \mathscr{U}(\mathscr{A}))$. Now, we set

$$
\begin{aligned}
\mu^{\prime}(m, n) & =\mu(m, n), \quad m, n \in N ; \\
\lambda^{\prime}(n,(g, t)) & =\alpha_{g}\left(\bar{b}\left(g^{-1} n g, t\right)\right) \lambda(n, g), \quad g \in G, t \in \mathbf{R} .
\end{aligned}
$$

Clearly, $\left(\lambda^{\prime}, \mu^{\prime}\right)$ extends $(\lambda, \mu)$. We want to show that $\left(\lambda^{\prime}, \mu^{\prime}\right)$ satisfies the conditions for $Z_{\alpha}(G \times \mathbf{R}, N, \mathscr{U}(\mathscr{A})),(1.6)-(1.9)$. But (1.6) is obvious. To check (1.7), let $H$ be the set of all $(g, t) \in G \times \mathbf{R}$ such 
that

$$
\begin{aligned}
& \alpha_{(g, t)}\left(\lambda^{\prime}\left((g, t)^{-1} n(g, t), h\right) \lambda^{\prime}(n,(g, t))\right) \\
& \quad=\lambda^{\prime}(n,(g, t) h), \quad n \in N, h \in G \times \mathbf{R} .
\end{aligned}
$$

If $h_{1}, h_{2} \in H$, we have

$$
\begin{aligned}
\alpha_{h_{1} h_{2}} & \left(\lambda^{\prime}\left(h_{2}^{-1} h_{1}^{-1} n h_{1} h_{2}, h\right)\right)=\alpha_{h_{1}}\left(\lambda^{\prime}\left(h_{1}^{-1} n h_{1}, h_{2} h\right) \lambda^{\prime}\left(h_{1}^{-1} n h_{1}, h_{2}\right)^{*}\right) \\
& =\lambda^{\prime}\left(n, h_{1} h_{2} h\right) \lambda^{\prime}\left(n, h_{1}\right)^{*} \lambda^{\prime}\left(n, h_{1} h_{2}\right)^{*} \lambda^{\prime}\left(n, h_{1}\right) \\
& =\lambda^{\prime}\left(n, h_{1} h_{2} h\right) \lambda^{\prime}\left(n, h_{1} h_{2}\right)^{*},
\end{aligned}
$$

so that $h_{1} h_{2} \in H$. To see $\mathbf{R} \subseteq H$, we compute:

$$
\begin{aligned}
\alpha_{t}\left(\lambda^{\prime}\right. & \left.\left((e, t)^{-1} n(e, t),(g, s)\right)\right) \lambda^{\prime}(n, t) \\
& =\alpha_{t}\left(\lambda^{\prime}(n,(g, s))\right) \bar{b}(n, t) \\
& =\alpha_{t}\left(\alpha_{g}\left(\bar{b}\left(g^{-1} n g, s\right)\right) \lambda(n, g)\right) \bar{b}(n, t) \\
& =\alpha_{t}\left(\alpha_{s}(\lambda(n, g)) \bar{b}(n, s)\right) \bar{b}(n, t) \\
& =\alpha_{s+t}(\lambda(n, g)) \bar{b}(n, s+t) \quad\left(\text { since } \bar{b}(n, \cdot) \in Z_{\alpha}^{1}(\mathbf{R}, \mathscr{U}(\mathscr{A}))\right), \\
& =\lambda^{\prime}(n,(g, s+t)) .
\end{aligned}
$$

Similarly, $G \subseteq H$ because for $g, h \in G$ and $s \in \mathbf{R}$,

$$
\begin{aligned}
& \alpha_{g}\left(\lambda^{\prime}\left(g^{-1} n g,(h, s)\right)\right) \lambda(n, g) \\
& \quad=\alpha_{g}\left(\alpha_{h}\left(\bar{b}\left(h^{-1} g^{-1} n g h, s\right)\right) \lambda\left(g^{-1} n g, h\right)\right) \lambda(n, g) \\
& \quad=\alpha_{g h}\left(\bar{b}\left(h^{-1} g^{-1} n g h, s\right)\right) \lambda(n, g h)=\lambda^{\prime}(n,(g h, s)) .
\end{aligned}
$$

Thus, $H=G \times \mathbf{R}$, and (1.7) for $\lambda^{\prime}$ follows.

To prove (1.9), we consider the set $K$ of all $(g, t) \in G \times \mathbf{R}$ such that

$$
\begin{aligned}
& \lambda^{\prime}(m,(g, t)) \lambda^{\prime}(n,(g, t)) \lambda^{\prime}(m n,(g, t))^{*} \\
& \quad=\alpha_{(g, t)}\left(\mu\left(g^{-1} m g, g^{-1} n g\right)\right) \mu(m, n)^{*}, \quad m, n \in N .
\end{aligned}
$$

Then a similar calculation shows that $K$ is closed under multiplication and contains $G$ and $\mathbf{R}$, so that (1.9) for $\left(\lambda^{\prime}, \mu^{\prime}\right)$ follows.

Therefore, $\operatorname{ker}(\gamma)$ is precisely the image of $\Lambda_{\alpha}(G \times \mathbf{R}, N, \mathscr{U}(\mathscr{A}))$ under the restriction map.

Exactness at $\Lambda_{\alpha}(G \times \mathbf{R}, N, \mathscr{U}(\mathscr{A}))$ : Suppose that $c$ is a $G$-equivariant homomorphism of $N$ into $Z_{\alpha}^{1}(\mathbf{R}, \mathscr{U}(\mathscr{A}))$. Set

$$
\mu^{\prime}=1 \quad \text { and } \quad \lambda^{\prime}(n,(g, s))=c(n, s), \quad n \in N, s \in \mathbf{R} .
$$


Then (1.6), (1.8), and (1.9) for $\left(\lambda^{\prime}, \mu^{\prime}\right)$ are obvious. For (1.7), we check:

$$
\begin{aligned}
\alpha_{(g, s)} & \left(\lambda^{\prime}\left(g^{-1} n g,(h, t)\right)\right) \lambda^{\prime}(n,(g, s)) \\
& =\alpha_{s} \alpha_{g}\left(c\left(g^{-1} n g, t\right)\right) c(n, s)=\alpha_{s}(c(n, t)) c(n, s) \\
& =c(n, s+t)=\lambda^{\prime}(n,(g h, s+t))=\lambda^{\prime}(n,(g, s)(h, t)) .
\end{aligned}
$$

Thus $\left(\lambda^{\prime}, \mu^{\prime}\right) \in Z_{\alpha}(G \times \mathbf{R}, N, \mathscr{U}(\mathscr{A}))$. We set

$$
j(c)=\left[\left(\lambda^{\prime}, \mu^{\prime}\right)\right] \in \Lambda_{\alpha}(G \times \mathbf{R}, N, \mathscr{U}(\mathscr{A})) .
$$

It is obvious that the restriction of $\left(\lambda^{\prime}, \mu^{\prime}\right)$ to $(G, N)$ gives a trivial element.

Suppose that $\left(\lambda^{\prime}, \mu^{\prime}\right) \in Z_{\alpha}(G \times \mathbf{R}, N, \mathscr{U}(\mathscr{A}))$ gives a trivial restriction to $(G, N)$. Then the class $\left[\lambda^{\prime}, \mu^{\prime}\right]$ is represented by a cocycle $\left(\lambda^{\prime}, \mu^{\prime}\right) \in Z_{\alpha}(G \times \mathbf{R}, N, \mathscr{U}(\mathscr{A}))$ such that $\mu^{\prime}(m, n)=1$ and $\lambda^{\prime}(n, g)=1$. Let $c(n, s)=\lambda^{\prime}(n, s), n \in N, s \in \mathbf{R}$. Then (1.7) and (1.9) for $\lambda^{\prime}$ imply that the map: $n \in N \rightarrow \lambda^{\prime}(n, \cdot) \in Z_{\alpha}^{1}(\mathbf{R}, \mathscr{U}(\mathscr{A}))$ is a $G$-equivariant homomorphism. Hence $c \in \operatorname{Hom}\left(N, Z_{\alpha}^{1}(\mathbf{R}, \mathscr{U}(\mathscr{A}))\right)^{G}$. By construction, $j(c)=\left[\lambda^{\prime}, \mu^{\prime}\right]$.

Exactness at $\operatorname{Hom}\left(N, Z_{\alpha}^{1}(\mathbf{R}, \mathscr{U}(\mathscr{A}))\right)^{G}$ : Let $b \in \operatorname{Hom}(N, \mathscr{U}(\mathscr{A}))^{G}$. That is, $b(m n)=b(m) b(n)$ and $\alpha_{g}\left(b\left(g^{-1} n g\right)\right)=b(n)$. Let $c(n, t)=$ $b(n)^{*} \alpha_{t}(b(n))$. Then $c(n, \cdot) \in Z_{\alpha}^{1}(\mathbf{R}, \mathscr{U}(\mathscr{A}))$ and $c$ is a $G$-equivariant homomorphism of $N$ into $Z_{\alpha}^{1}(\mathbf{R}, \mathscr{U}(\mathscr{A}))$. We put $c=\partial b$. Then $\partial$ is a homomorphism of $\operatorname{Hom}(N, \mathscr{U}(\mathscr{A}))^{G}$ to $\operatorname{Hom}\left(N, Z_{\alpha}^{1}(\mathbf{R}, \mathscr{U}(\mathscr{A}))\right)^{G}$. If $\left[\lambda^{\prime}, \mu^{\prime}\right]=j(c)$, then $\mu^{\prime}=1$ and

$$
\begin{aligned}
\lambda^{\prime}(n,(g, t)) & =c(n, t)=b(n)^{*} \alpha_{t}(b(n)) \\
& =\alpha_{t} \alpha_{g}\left(b(g, t)^{-1} n(g, t)\right) b(n)^{*} \\
& =\left(\partial_{2} b\right)(n,(g, t)) .
\end{aligned}
$$

Thus we get $\left[\lambda^{\prime}, \mu^{\prime}\right]=1$. Hence $\operatorname{Im}(\partial) \subseteq \operatorname{ker}(j)$.

Conversely, suppose $j(c)=1, c \in \operatorname{Hom}\left(N, Z_{\alpha}^{1}(\mathbf{R}, \mathscr{U}(\mathscr{A}))\right)^{G}$. Namely, with $\mu^{\prime}=1$ and $\lambda^{\prime}(n, g, t)=c(n, t)$, we have $\left(\lambda^{\prime}, \mu^{\prime}\right) \in$ $B_{\alpha}(G \times \mathbf{R}, N, \mathscr{U}(\mathscr{A}))$. Hence there exists $b \in \operatorname{Map}(N, \mathscr{U}(\mathscr{A}))$ such that

$$
\begin{aligned}
b(n) b(m) b(n m)^{*} & =\partial_{1} b(n, m)=1 ; \\
\alpha_{t} \alpha_{g}\left(b\left(g^{-1} n g\right)\right) b(n)^{*} & =\partial_{2} b(n,(g, t)) \\
& =\lambda^{\prime}(n,(g, t))=c(n, t) .
\end{aligned}
$$

The first identity shows that $b \in \operatorname{Hom}(N, \mathscr{U}(\mathscr{A}))$. In the second identity, with $t=0$, we get $\alpha_{g}\left(b\left(g^{-1} n g\right)\right)=b(n)$. Hence $b \in$ $\operatorname{Hom}(N, \mathscr{U}(\mathscr{A}))^{G}$ and $c=\partial b$. 
We now move on to the second part of Theorem 2.2. Given $\chi^{\prime}=$ $\left[\lambda^{\prime}, \mu^{\prime}\right] \in \Lambda_{\alpha}(G \times \mathbf{R}, N, \mathscr{U}(\mathscr{A}))$, we studied the restriction $(\lambda, \mu)$ of $\left(\lambda^{\prime}, \mu^{\prime}\right)$ to $(G, N)$. But the restriction $c$ of $\lambda^{\prime}$ to $N \times \mathbf{R}$ gives an element of $\operatorname{Hom}\left(N, H_{\alpha}^{1}(\mathbf{R}, \mathscr{U}(\mathscr{A}))\right)^{G}$. In fact, (1.9) for $\lambda^{\prime}$ shows that the map $c: n \in N \rightarrow \lambda^{\prime}(n, \cdot) \in Z_{\alpha}^{1}(\mathbf{R}, \mathscr{U}(\mathscr{A}))$ is multiplicative up to cohomology. Furthermore, the calculation of Lemma 2.1 shows that

$$
\left(\alpha_{g}\left(\lambda^{\prime}\right) \lambda^{\prime *}\right)(n, t)=\left(\alpha_{t}\left(\lambda^{\prime}\right) \lambda^{\prime *}\right)(n, g),
$$

so that $c$ is $G$-equivariant up to cohomology. Hence the map $\nu: n \in$ $N \rightarrow[c(n)] \in H_{\alpha}^{1}(\mathbf{R}, \mathscr{U}(\mathscr{A}))$ is a $G$-equivariant homomorphism.

Now, suppose $\chi=[\lambda, \mu] \in \Lambda_{\alpha}(G, N, \mathscr{U}(\mathscr{A}))^{\mathbf{R}}$. We define

$$
\begin{gathered}
\tilde{\lambda}(t, n, g)=\left(\alpha_{t}(\lambda) \lambda^{*}\right)(n, g)=\alpha_{t}(\lambda(n, g)) \lambda(n, g)^{*} \\
\tilde{\mu}(t, m, n)=\left(\alpha_{t}(\mu) \mu^{*}\right)(m, n)=\alpha_{t}(\mu(m, n)) \mu(m, n)^{*}
\end{gathered}
$$

for $t \in \mathbf{R}, m, n \in N$ and $g \in G$. Since $\alpha_{t}(\chi)=\chi,(\tilde{\lambda}(t, \cdot, \cdot), \tilde{\mu}(t, \cdot, \cdot))$ is an element of $B_{\alpha}(G, N, \mathscr{U}(\mathscr{A}))$, and hence gives an element of $Z_{\alpha}^{1}\left(\mathbf{R}, B_{\alpha}(G, N, \mathscr{U}(\mathscr{A}))\right)$.

LEMMA 2.5. The cohomology class $[\tilde{\lambda}, \tilde{\mu}] \in H_{\alpha}^{1}\left(\mathbf{R}, B_{\alpha}(G, N, \mathscr{U}(\mathscr{A}))\right)$ depends only on $\chi$; we write it as $\delta_{1}(\chi)$. The map

$$
\delta_{1}: \chi \in \Lambda_{\alpha}(G, N, \mathscr{U}(\mathscr{A}))^{\mathbf{R}} \rightarrow \delta_{1}(\chi) \in H_{\alpha}^{1}\left(\mathbf{R}, B_{\alpha}(G, N, \mathscr{U}(\mathscr{A}))\right)
$$

is a homomorphism.

Proof. The map $(\lambda, \mu) \rightarrow(\tilde{\lambda}, \tilde{\mu})$ is multiplicative, so that it suffices to show that if $(\lambda, \mu) \in B_{\alpha}(G, N, \mathscr{U}(\mathscr{A}))$, then $[\tilde{\lambda}, \tilde{\mu}]=1$. But if $(\lambda, \mu) \in$ $B_{\alpha}(G, N, \mathscr{U}(\mathscr{A}))$, then

$$
(\tilde{\lambda}, \tilde{\mu})(t, \cdot, \cdot)=\alpha_{t}((\lambda, \mu)(\lambda, \mu))^{*},
$$

which means that $[\tilde{\lambda}, \tilde{\mu}]=1$ in $H_{\alpha}^{1}\left(\mathbf{R}, B_{\alpha}(G, N, \mathscr{U}(\mathscr{A}))\right)$.

Next, suppose $\nu \in \operatorname{Hom}\left(N, H_{\alpha}^{1}(\mathbf{R}, \mathscr{U}(\mathscr{A}))\right)$, and let $c: n \in N \rightarrow$ $c(n) \in Z_{\alpha}^{1}(\mathbf{R}, \mathscr{U}(\mathscr{A}))$ be a Borel map which projects to $\nu$. Consider $\left(\partial_{2} c(\cdot, t), \partial_{1} c(\cdot, t)\right)$ as an element of $Z_{\alpha}^{1}\left(\mathbf{R}, B_{\alpha}(G, N, \mathscr{U}(\mathscr{A}))\right)$.

LEMMA 2.6. The cohomology class $\left[\partial_{2} c, \partial_{1} c\right]$ in

$$
H_{\alpha}^{1}\left(\mathbf{R}, B_{\alpha}(G, N, \mathscr{U}(\mathscr{A}))\right)
$$

depends only on $\nu$; we write it $\delta_{2}(\nu)$. Of course, the map

$$
\delta_{2}: \operatorname{Hom}\left(N, H_{\alpha}^{1}(\mathbf{R}, \mathscr{U}(\mathscr{A}))\right) \rightarrow H_{\alpha}^{1}(\mathbf{R}, B(G, N, \mathscr{U}(\mathscr{A})))
$$

is a homomorphism. 
Proof. Since the map: $c \rightarrow\left(\partial_{2} c, \partial_{1} c\right)$ is multiplicative, it suffices to prove that if $c(n, \cdot) \in B_{\alpha}^{1}(\mathbf{R}, \mathscr{U}(\mathscr{A}))$, then $\left[\partial_{2} c, \partial_{1} c\right]=1$. Choose a Borel map $b: N \rightarrow \mathscr{U}(\mathscr{A})$ so that $c(n, t)=\alpha_{t}(b(n)) b(n)^{*}$. Then we have $\left(\partial_{2} c, \partial_{1} c\right)(t)=\alpha_{t}\left(\partial_{2} b, \partial_{1} b\right)\left(\partial_{2} b^{*}, \partial_{1} b^{*}\right)$, so that $\left[\partial_{2} c, \partial_{1} c\right]=1$.

Proof of Theorem 2.2 (ii). Exactness at $\Lambda_{\alpha}(G, N, \mathscr{U}(\mathscr{A}))^{\mathbf{R}} \times$ $\operatorname{Hom}\left(N, H_{\alpha}^{1}(\mathbf{R}, \mathscr{U}(\mathscr{A}))\right)$ : We define

$$
\delta(\chi, \nu)=\delta_{1}(\chi)-\delta_{2}(\nu)
$$

for $(\chi, \nu) \in \Lambda_{\alpha}(G, N, \mathscr{U}(\mathscr{A}))^{\mathbf{R}} \times \operatorname{Hom}\left(N, H_{\alpha}^{1}(\mathbf{R}, \mathscr{U}(\mathscr{A}))\right)$. If $\chi^{\prime}=\left[\lambda^{\prime}, \mu^{\prime}\right]$ $\in \Lambda_{\alpha}(G \times \mathbf{R}, N, \mathscr{U}(\mathscr{A}))$ gives rise to $(\chi, \nu)$ as restriction, (2.1), (2.2) and $\left(2.2^{\prime}\right)$ mean that $\delta_{1}(\chi)=\delta_{2}(\nu)$. Conversely, suppose that $\delta_{1}(\chi)=$ $\delta_{2}(\nu)$. Choose $(\lambda, \mu) \in Z_{\alpha}(G, N, \mathscr{U}(\mathscr{A}))$ with $\chi=[\lambda, \mu]$ and $c: N \times \mathbf{R} \rightarrow$ $c(n, t) \in \mathscr{U}(\mathscr{A})$ lifting $\nu$. Choose $b: n \in N \rightarrow b(n) \in \mathscr{U}(\mathscr{A})$ so that

$$
\begin{aligned}
\left(\alpha_{t}(\lambda) \lambda^{*}\right)(n, g)\left(\alpha_{t}\left(\partial_{2} b\right) \partial_{2} b^{*}\right)(n, g) & =\left(\partial_{2} c\right)(n, g, t), \\
\left(\alpha_{t}(\mu) \mu^{*}\right)(m, n)\left(\alpha_{t}\left(\partial_{1} b\right) \partial_{2} b^{*}\right)(m, n) & =\left(\partial_{1} c\right)(m, n)
\end{aligned}
$$

for $m, n \in N, g \in G$ and $t \in \mathbf{R}$. Replacing $(\lambda, \mu)$ by $\left(\lambda \partial_{2} b, \mu \partial_{1} b\right)$, we get

$$
\begin{aligned}
\alpha_{t}(\lambda(n, g)) \lambda(n, g)^{*} & =\alpha_{g}\left(c\left(g^{-1} n g, t\right)\right) c(n, t)^{*}, \\
\alpha_{t}(\mu(m, n)) \mu(m, n)^{*} & =c(m, t) c(n, t) c(m n, t)^{*} .
\end{aligned}
$$

We then extend $(\lambda, \mu)$ to $\left(\lambda^{\prime}, \mu^{\prime}\right) \in Z_{\alpha}(G \times \mathbf{R}, N, \mathscr{U}(\mathscr{A}))$ by setting

$$
\begin{gathered}
\lambda^{\prime}(n, g, t)=\alpha_{t}(\lambda(n, g)) c(n, t), \quad g \in G, t \in \mathbf{R} ; \\
\mu^{\prime}(m, n)=\mu(m, n), \quad m, n \in N,
\end{gathered}
$$

where the cocycle property of $\left(\lambda^{\prime}, \mu^{\prime}\right)$ can be checked by applying the arguments of the second paragraph of the proof of Theorem 2.2 (i).

Exactness at $\Lambda_{\alpha}(G \times \mathbf{R}, N, \mathscr{U}(\mathscr{A}))$. Let $\phi \in \operatorname{Hom}(N, \mathscr{U}(\mathscr{A}) / \mathbf{T})^{G}$. Choose a map $b: n \in N \rightarrow b(n) \in \mathscr{U}(\mathscr{A})$ and $\sigma: N \times N \rightarrow$ T such that $\phi(n)=b(n) \mathbf{T}$ and

$$
b(m) b(n)=\sigma(m, n) b(m, n), \quad m, n \in N .
$$

Since $\phi$ is $G$-equivariant, there exists a function $\rho: N \times G \rightarrow \mathrm{T}$ so that

$$
\alpha_{g}\left(b\left(g^{-1} n g\right)\right)=\rho(n, g) b(n), \quad g \in G, n \in N .
$$

Clearly $(\rho, \sigma) \in Z(G, N, \mathbf{T})$. Set

$$
c(n, s)=\alpha_{s}(b(n)) b(n)^{*}, \quad n \in N, s \in \mathbf{R},
$$


and compute:

$$
\begin{aligned}
c(m, s) c(n, s) & =\alpha_{s}(b(m)) b(n) b(m)^{*} b(n)^{*} \\
& =\alpha_{s}(\sigma(m, n) b(m n)) \overline{\sigma(m, n)} b(m n)^{*}=c(m n, s) \\
\alpha_{g}\left(c\left(g^{-1} n g, s\right)\right) & =\alpha_{s}\left(\alpha_{g}\left(b\left(g^{-1} n g\right)\right)\right) \alpha_{g}\left(b\left(g^{-1} n g\right)^{*}\right) \\
& =\alpha_{s}(\rho(n, g) b(n)) \overline{\rho(n, g)} b(n)^{*}=c(n, s) .
\end{aligned}
$$

Thus, we obtain $c \in \operatorname{Hom}\left(N, B_{\alpha}^{1}(\mathbf{R}, \mathscr{U}(\mathscr{A}))\right)^{G}$. We now apply the homomorphism $j$ of $\operatorname{Hom}\left(N, Z^{1}(\mathbf{R}, \mathscr{U}(\mathscr{A}))\right)^{G}$ into $\Lambda_{\alpha}(G \times \mathbf{R}, N, \mathscr{U}(\mathscr{A}))$ in (2.3) to get $\left[\lambda^{\prime}, \mu^{\prime}\right]=j(c) \in \Lambda_{\alpha}(G \times \mathbf{R}, N, \mathscr{U}(\mathscr{A}))$. We have seen in (i) that $\left[\left.\left(\lambda^{\prime}, \mu^{\prime}\right)\right|_{(G, N)}\right]=1$. It is also clear that $\left.\lambda^{\prime}\right|_{N \times \mathbf{R}}=1$ in $\operatorname{Hom}\left(N, H_{\alpha}^{1}(\mathbf{R}, \mathscr{U}(\mathscr{A}))\right)$.

Suppose that $\left[\lambda^{\prime}, \mu^{\prime}\right]=\chi^{\prime} \in \Lambda_{\alpha}(G \times \mathbf{R}, N, \mathscr{U}(\mathscr{A}))$ has trivial restriction to $(G, N)$ and $N \times \mathbf{R}$. Then $\chi^{\prime}$ is represented by

$$
\left(\lambda^{\prime}, \mu^{\prime}\right) \in Z_{\alpha}(G \times \mathbf{R}, N, \mathscr{U}(\mathscr{A}))
$$

such that $\mu^{\prime}=1$ and $\lambda^{\prime}(n, g)=1, n \in N$ and $g \in G$. With $c(n, s)=$ $\lambda^{\prime}(n, s)$, as in (i) $c$ gives an element of $\operatorname{Hom}\left(N, Z_{\alpha}^{1}(\mathbf{R}, \mathscr{U}(\mathscr{A}))\right)^{G}$. But $c(n, \cdot) \in B_{\alpha}^{1}(\mathbf{R}, \mathscr{U}(\mathscr{A}))$ due to the triviality of $\left.\lambda^{\prime}\right|_{N \times \mathbf{R}}$, so that there exists a map $b: n \in N \rightarrow b(n) \in \mathscr{U}(\mathscr{A})$ such that $c(n, s)=\alpha_{s}(b(n)) b(n)^{*}$. Since $\mathbf{R}$ acts ergodically, the ambiguity in choosing $b(n)$ is precisely $\mathbf{T}$, so that the map $b: n \in N \rightarrow b(n) \in \mathscr{U}(\mathscr{A})$ is a homomorphism up to phase, i.e. $\phi(n)=b(n) \mathbf{T} \in \mathscr{U}(\mathscr{A}) / \mathbf{T}$ is a homomorphism. It is now clear that $\phi \in \operatorname{Hom}(N, \mathscr{U}(\mathscr{A}) / \mathbf{T})^{G}$ and the image of $\phi$ in $\Lambda_{\alpha}(G \times \mathbf{R}, N, \mathscr{U}(\mathscr{A}))$ is precisely $\chi^{\prime}$.

Exactness at $\operatorname{Hom}(N, \mathscr{U}(\mathscr{A}) / \mathbf{T})^{G}$ : The natural projection of $\mathscr{U}(\mathscr{A})$ onto $\mathscr{U}(\mathscr{A}) / \mathrm{T}$ gives rise to a homomorphism of $\operatorname{Hom}(N, \mathscr{U}(\mathscr{A}))^{G}$ into $\operatorname{Hom}(N, \mathscr{U}(\mathscr{A}) / \mathbf{T})^{G}$. The image of this homomorphism are precisely liftable homomorphism $\phi \in \operatorname{Hom}(N, \mathscr{U}(\mathscr{A}) / \mathbf{T})^{G}$, i.e. those $\phi$ such that the map $b$ in the last paragraph is a $G$-equivariant homomorphism; equivalently $\rho=1$ and $\sigma=1$. Then the $c$ in (2.20) is in the image of $\operatorname{Hom}(N, \mathscr{U}(\mathscr{A}))^{G}$ under the homomorphism in (2.3). Hence the image of $\phi$ in $\Lambda_{\alpha}(G \times \mathbf{R}, N, \mathscr{U}(\mathscr{A}))$ is the identity.

Conversely, suppose that $\phi \in \operatorname{Hom}(N, \mathscr{U}(\mathscr{A}) / \mathbf{T})^{G}$ gives rise to the identity element of $\Lambda_{\alpha}(G \times \mathbf{R}, N, \mathscr{U}(\mathscr{A}))$. Then the $c$ in (2.20) is given by an element $b^{\prime} \in \operatorname{Hom}(N, \mathscr{U}(\mathscr{A}))^{G}$ in such a way that

$$
c(n, t)=\alpha_{t}\left(b^{\prime}(n)\right) b^{\prime}(n)^{*} .
$$

Hence with $b$ as in the last paragraph, we have

$$
\alpha_{t}(b(n)) b(n)^{*}=\alpha_{t}\left(b^{\prime}(n)\right) b^{\prime}(n)^{*},
$$


so that the ergodicity of $\mathbf{R}$ on $\mathscr{A}$ implies that $b(n) b^{\prime}(n)^{*} \in \mathbf{T}$. Hence we have $\phi(n)=b^{\prime}(n) \mathbf{T}$. Thus $\phi$ is given as the projection of $b^{\prime}$ in $\mathscr{U}(\mathscr{A}) / \mathbf{T}$.

Proof of Theorem 2.2 (iii). We first construct the injection of $\Lambda(G, N, \mathbf{T})$ in $\Lambda_{\alpha}(G \times \mathbf{R}, N, \mathscr{U}(\mathscr{A}))$. If $\chi=[\lambda, \mu] \in \Lambda(G, N, \mathbf{T})$, the arguments of the proof of 2.2 (i) show that there is an element $\left(\lambda^{\prime}, \mu^{\prime}\right) \in$ $Z_{\alpha}(G \times \mathbf{R}, N, \mathscr{U}(\mathscr{A}))$ with $\mu^{\prime}(m, n)=\mu(m, n), \lambda^{\prime}(n, g)=\lambda(n, g)$ and $\lambda^{\prime}(n, t)=1$ for $m, n \in N, g \in G$ and $t \in \mathbf{R}$ (see equation (2.14)). It is evident that $\chi^{\prime}=\left[\lambda^{\prime}, \mu^{\prime}\right]$ depends only on $\chi$ and that the map $\chi \rightarrow \chi^{\prime}$ is a homomorphism. To see that it is injective, suppose $(\lambda, \mu) \in Z(G, N, \mathbf{T})$ is such that $\left(\lambda^{\prime}, \mu^{\prime}\right) \in Z_{\alpha}(G \times \mathbf{R}, N, \mathscr{U}(\mathscr{A}))$ constructed as above, cobounds. Thus there is a map $b: N \rightarrow \mathscr{U}(\mathscr{A})$ such that $\left(\lambda^{\prime}, \mu^{\prime}\right)=\left(\partial_{2} b, \partial_{1} b\right)$. Thus $1=\lambda^{\prime}(n, t)=\alpha_{t}(b(n)) b(n)^{*}$ for $n \in N$ and $t \in \mathbf{R}$, so that, using ergodicity of $\mathbf{R}, b(n) \in \mathbf{T}$. It now follows that $(\lambda, \mu) \in B(G, N, \mathbf{T})$, as required.

It remains to prove exactness at $\Lambda(G \times \mathbf{R}, N, \mathscr{U}(\mathscr{A}))$. It is clear that $\Lambda(G, N, \mathbf{T}) \subseteq \operatorname{ker} r$. Conversely, suppose $\chi^{\prime}=\left[\lambda^{\prime}, \mu^{\prime}\right] \in \operatorname{ker} r$. By definition, there is a map $b: N \rightarrow \mathscr{U}(\mathscr{A})$ such that $\lambda^{\prime}(n, t)=\alpha_{t}(b(n)) b(n)^{*}$. However, since $\lambda^{\prime}(n, g t)=\lambda^{\prime}(n, t g)$, we conclude using (1.7) that

$$
\alpha_{t}\left(\alpha_{g}\left(b\left(g^{-1} n g\right)\right) b(n)^{*} \lambda^{\prime}(n, g)\right)=\alpha_{g}\left(b\left(g^{-1} n g\right)\right) b(n)^{*} \lambda^{\prime}(n, g) .
$$

Since $\mathbf{R}$ acts ergodically, we conclude that $\alpha_{g}\left(b\left(g^{-1} n g\right)\right) b(n)^{*} \lambda^{\prime}(n, g)=$ $\kappa(n, g) \in \mathbf{T}$. Similarly, using (1.9) with $g=t \in \mathbf{R}$, we conclude $b(m)^{*} b(n)^{*} b(m, n) \mu(m, n)=\sigma(m, n) \in \mathbf{T}$. Thus $\left(\lambda^{\prime} \partial_{2} b^{*}, \mu^{\prime} \partial_{1} b^{*}\right)=$ $\left(\kappa^{\prime}, \sigma^{\prime}\right) \in Z(G \times \mathbf{R}, N, \mathbf{T})$, and since $\kappa^{\prime}(n, t)=1,\left[\lambda^{\prime}, \mu^{\prime}\right]=\left[\kappa^{\prime}, \sigma^{\prime}\right]$ is in the image of $\lambda(G, N, \mathbf{T})$.

3. Reduction and $C^{\infty}$-representatives of $H_{\alpha}^{1}(\mathbf{R}, \mathscr{U}(\mathscr{A}))$. In this section, we consider a strongly continuous one parameter automorphism group $\left\{\alpha_{t}\right\}$ on an abelian von Neumann algebra $\mathscr{A}$. Here we assume that $\left\{\alpha_{t}\right\}$ is ergodic and aperiodic, i.e. free. By the Ambrose-KakutaniKubo-Krengel theorem, we may assume that the point realization $\left\{T_{t}\right\}$ on $\left\{X, m^{\prime}\right\}$ of $\left\{\alpha_{t}\right\}$ on $\mathscr{A}$ is the flow built over the base $\{B, m, Q\}$ under the ceiling function $f: B \rightarrow \mathbf{R}_{+}$where $f$ is a Borel function bounded away from zero. Thus, $X$ is identified with the quotient of $B \times \mathbf{R}$ by the action of $Z$ generated by the transformation $\tilde{Q}$ :

$$
\tilde{Q}(b, r)=(Q b, r-f(b)), \quad b \in B, r \in \mathbf{R},
$$

i.e. with $\{(n, r) \in B \times \mathbf{R}: 0 \leq r<f(b)\}$, and the measure $m^{\prime}$ is identified with the restriction of $m \times$ Lebesgue to this fundamental 
domain. The flow $\left\{T_{t}\right\}$ is determined by the flow:

$$
\tau_{s}(b, r)=(b, r+s) \text { on } X \times \mathbf{R} .
$$

Let $p:(b, r) \in X \rightarrow b \in B$ be the projection.

We will view $\mathscr{G}^{\prime}=\mathbf{R} \ltimes X$ and $\mathscr{G}=\left.\mathscr{G}^{\prime}\right|_{B}=Z \ltimes_{Q} B$ as groupoids. Since we are assuming $\left\{T_{t}\right\}$ acts freely, both $\mathscr{G}^{\prime}$ and $\mathscr{G}$ may be viewed as equivalence relations.

To each $A \in Z^{1}(\mathscr{G}, \mathbf{T})$, there corresponds $p^{*}(A) \in Z^{1}\left(\mathscr{G}^{\prime}, \mathbf{T}\right)$ by

$$
\left(p^{*} A\right)\left(T_{t} x, x\right)=A\left(p\left(T_{t} x\right), p(x)\right), \quad x \in X, t \in \mathbf{R} .
$$

Also if $A \in Z^{1}\left(\mathscr{G}^{\prime}, \mathbf{T}\right)$, so that

$$
A\left(T_{s+t} x, x\right)=A\left(T_{s+t} x, T_{t} x\right) A\left(T_{t} x, x\right)
$$

almost everywhere in $x$ for each $s, t \in \mathbf{R}$, we may define $L^{\infty}(A)(t) \in$ $L^{\infty}\left(X, m^{\prime}\right)$ to be the element represented by the function $x \in X \rightarrow$ $A\left(T_{t} x, x\right)$. Evidently, $L^{\infty}(A) \in Z_{\alpha}^{1}(\mathbf{R}, \mathscr{U}(\mathscr{A}))$. The maps $p^{*}$ and $L^{\infty}$ give rise to maps, also denoted by $p^{*}$ and $L^{\infty}$, from $H^{1}(\mathscr{G}, \mathbf{T})$ to $H^{1}\left(\mathscr{G}^{\prime}, \mathbf{T}\right)$ and from $H^{1}\left(\mathscr{G}^{\prime}, \mathbf{T}\right)$ to $H_{\alpha}^{1}(\mathbf{R}, \mathscr{U}(\mathscr{A}))$. The following result was proved in [4, Proposition A.2]. Since the proof given there is slightly imprecise in handling null sets, and the notations will be needed later anyway, we present a proof.

THEOREM 3.1. The maps $p^{*}: H^{1}(\mathscr{G}, \mathbf{T}) \rightarrow H^{1}\left(\mathscr{G}^{\prime}, \mathbf{T}\right)$ and $L^{\infty}$ : $H^{1}\left(\mathscr{G}^{\prime}, \mathbf{T}\right) \rightarrow H_{\alpha}^{1}(\mathbf{R}, \mathscr{U}(\mathscr{A}))$ are isomorphisms.

Proof. It suffices to prove that $L^{\infty} \circ p^{*}$ is an isomorphism. Let $a \in Z_{\alpha}^{1}(\mathbf{R}, \mathscr{U}(\mathscr{A}))$. Since $\mathscr{A}$ may be identified with the fixed point algebra $L^{\infty}(B \times \mathbf{R})^{\tilde{Q}}$ of $L^{\infty}(B \times \mathbf{R})$ under the action determined by $\tilde{Q}$, and $(B, m)$ is Borel isomorphic to ( $\mathbf{T}$, Lebesgue), we apply the equivariant lifting theorem of [6] with the group $\mathbf{T} \times \mathbf{R}$ and obtain a measurable function $A: \mathbf{R} \times B \times \mathbf{R} \rightarrow \mathbf{T}$ with the properties:

(i) $A(s+t, b, r)=A(s, b, r) A(t, b, r-s)$ for all $r, s, t \in \mathbf{R}$ and $b \in B$;

(ii) for each $s \in \mathbf{R}, A(s, \tilde{Q}(b, r))=A(s, b, r)$ almost everywhere in $(b, r)$;

(iii) for each $s \in \mathbf{R}, a(s)$ is represented by the function $(b, r) \rightarrow$ $A(s, b, r)$.

Define

$$
B\left(\tau_{s}(b, r),(b, r)\right)=A(s, b, r) ;
$$

from (i) above, $B \in Z^{1}\left(\mathbf{R} \ltimes_{\tau}(B \times \mathbf{R})\right.$, $\left.\mathbf{T}\right)$. 
Let $c: B \times \mathbf{R} \rightarrow \mathbf{T}$ be the $\tilde{Q}$-periodic function which extends the map $(b, r) \in X \rightarrow B((b, r),(b, 0))$ and define $D \in Z^{1}\left(\mathbf{R} \ltimes_{\tau}(B \times \mathbf{R}), \mathbf{T}\right)$ by

$$
D((b, r),(b, s))=c(b, r)^{-1} B((b, r),(b, s)) c(b, s) .
$$

By definition, $D$ is cohomologous to $B$ in $Z^{1}\left(\mathbf{R} \ltimes_{\tau}(B \times \mathbf{R})\right.$, T). By (ii) above, and since $c$ is $\tilde{Q}$-invariant, we see that for $0 \leq r, s<f(b)$,

$$
D\left(\tilde{Q}(b, r), \tilde{Q}^{n}(b, s)\right)=1
$$

almost everywhere in $(b, r, s)$. Thus, for almost every $b \in B$, the function: $(r, s) \in[0, f(Q b)[\times[0, f(b)[\rightarrow D((b, r),(b, s))$ is constant almost everywhere in $(r, s)$; we let $E^{\prime}(b)$ denote this constant value, and let $E \in Z^{1}(\mathscr{G}, \mathbf{T})$ be the unique cocycle with $E(Q b, b)=E^{\prime}(b)$.

It is now clear that if $c \in L^{\infty}\left(X, m^{\prime}\right)$ is represented by the above function $c$, then $L^{\infty}\left(p^{*} E\right)=\left(\partial c^{*}\right) a$ in $Z_{\alpha}^{1}(\mathbf{R}, \mathscr{U}(\mathscr{A}))$; we leave the routine verification to the reader.

THEOREM 3.2. Under the same assumptions and notations as in the previous theorem, every $a \in Z_{\alpha}^{1}(\mathbf{R}, \mathscr{U}(\mathscr{A}))$ is cohomologous to a cocycle which is infinitely differentiable in norm.

Proof. By the previous theorem, there is a cocycle $A \in Z^{1}\left(\mathbf{Z}_{\ltimes_{Q}} B, \mathbf{T}\right)$ such that $a$ is cohomologous to $L^{\infty}\left(p^{*} A\right)$. Let $C^{\prime}: B \rightarrow[0,1]$ be a Borel function such that

$$
A(Q b, b)=\exp \left(2 \pi i C^{\prime}(b)\right),
$$

and let $C \in Z^{1}\left(\mathbf{Z} \ltimes_{Q} B, \mathbf{R}\right)$ be the unique cocycle with $C(Q b, b)=C^{\prime}(b)$ for $b \in B$. Evidently, $\exp (2 \pi i C)=A$, so that $L^{\infty}\left(\exp \left(2 \pi i p^{*} C\right)\right)$ is cohomologous to $a$.

Choose a $C^{\infty}$-function $g:[0,1] \rightarrow \mathbf{R}_{+}$with $\int_{0}^{1} g(s) d s=1$, and such that $g$ is zero outside of $[1 / 3,2 / 3]$. For $b \in B$ and $0 \leq s<f(b)$, define

$$
g_{b}(s)=\frac{C(Q b, b)}{f(b)} g\left(\frac{s}{f(b)}\right)
$$

and

$$
G(b, r)=\int_{0}^{r} g_{b}(s) d s
$$

Set

$$
D\left(T_{t}(b, r),(b, r)\right)=G\left(T_{t}(b, r)\right)+C\left(p\left(T_{t}(b, r)\right), b\right)-G(b, r),
$$

so that $D$ is cohomologous in $Z^{1}\left(\mathbf{R} \ltimes_{T} X, \mathbf{R}\right)$ with $p^{*} C$. 
Using the properties demanded of $g$ and the fact that $f$ is bounded away from zero, it is easy to see that the function: $t \in \mathbf{R} \rightarrow D\left(T_{t} x, x\right)$ is $C^{\infty}$ for each $x \in X$, and that each derivative of this function is uniformly bounded in $t$ and $x$. From this, it follows that $L^{\infty}(\exp 2 \pi i D)$ is infinitely differentiable in norm as an element of $Z_{\alpha}^{1}(\mathbf{R}, \mathscr{U}(\mathscr{A}))$. Since this cocycle is cohomologous to $a$, the proof is complete.

4. Actions of $G \times \mathbf{R}$ on $\mathscr{A} \bar{\otimes} \mathscr{R}_{0,1}$. Throughout this section, $G$ denotes a countable discrete amenable group, $\mathscr{A}$ an abelian von Neumann algebra, and $\mathscr{R}_{0,1}$ the unique injective factor of type $\mathrm{II}_{\infty}$. We are concerned with cocycle conjugacy classification of actions of $G \times \mathbf{R}$ on $\mathscr{A} \bar{\otimes} \mathscr{R}_{0,1}$. Our technique does not apply to the case that $\mathscr{A}=\mathbf{C}$ and $G$ is trivial. Thus we need to make some assumptions; these assumptions will be sufficient to allow us, in $\S 5$, to classify actions of $G$ on injective factors of type $\mathrm{III}_{\lambda}, \lambda \neq 1$. Now, we make the following:

Assumption 4.1. (i) $\mathscr{A} \neq \mathbf{C}$, and $\left\{\alpha_{t}: t \in \mathbf{R}\right\}$ is ergodic on $\mathscr{A}$;

(ii) there is a faithful semi-finite normal trace $\operatorname{Tr}$ on $\mathscr{A} \bar{\otimes}_{0,1}$ such that

$$
\begin{gathered}
\operatorname{Tr} \circ \alpha_{g}=\operatorname{Tr}, \quad g \in G ; \\
\operatorname{Tr} \circ \alpha_{s}=e^{-s} \operatorname{Tr}, \quad s \in \mathbf{R} .
\end{gathered}
$$

Our strategy for classifying actions of $G \times \mathbf{R}$ satisfying our basic assumption is to show that the problem may be reduced to that of classifying actions of a discrete groupoid on $\mathscr{R}_{0,1}$, and to use results about such actions from [15].

\subsection{The invariants and the classification. Let}

$$
\alpha: G \times \mathbf{R} \rightarrow \operatorname{Aut}\left(\mathscr{A} \otimes \mathscr{R}_{0,1}\right)
$$

be an action satisfying our basic assumption. We may associate cocycle conjugacy invariants for $\alpha$ in the same way as is done for discrete groups. Let

$$
N(\alpha)=\left\{g \in G \times \mathbf{R}: \alpha_{g} \in \operatorname{Int}\left(\mathscr{A} \bar{\otimes} \mathscr{R}_{0,1}\right)\right\} .
$$

Note that our assumption concerning the trace guarantees that $N(\alpha) \triangleleft G$, and so is discrete and countable. Choose a (Borel) map: $n \in N(\alpha) \rightarrow u(n) \in \mathscr{U}\left(\mathscr{A} \otimes \mathscr{R}_{0,1}\right)$ such that

$$
\alpha_{n}=\operatorname{Ad}(u(n)), \quad n \in N(\alpha)
$$


and define $\mu_{\alpha}(m, n) \in \mathscr{U}(\mathscr{A})$ for $m, n \in N(\alpha)$ and $\lambda_{\alpha}(n, h) \in \mathscr{U}(\mathscr{A})$ for $n \in N(\alpha)$ and $h \in G \times \mathbf{R}$ by

$$
\begin{aligned}
u(m) u(n) & =\mu_{\alpha}(m, n) u(m n), \\
\alpha_{h}\left(u\left(h^{-1} n h\right)\right) & =\lambda_{\alpha}(n, h) u(n) .
\end{aligned}
$$

Observe that the pair $\left(\lambda_{\alpha}, \mu_{\alpha}\right)$ falls in $Z_{\alpha}(G \times \mathbf{R}, N(\alpha), \mathscr{U}(\mathscr{A}))$, and the class $\chi_{\alpha}$ of $\left(\lambda_{\alpha}, \mu_{\alpha}\right)$ in $\Lambda_{\alpha}(G \times \mathbf{R}, N(\alpha), \mathscr{U}(\mathscr{A}))$, depends only on the cocycle conjugacy class of $\alpha$ (see below for a more precise statement).

THEOREM 4.2. Let $\alpha$ and $\beta$ be actions of $G \times \mathbf{R}$ on $\mathscr{A} \otimes \mathscr{R}_{0,1}$ satisfying Assumption 4.1. Then the following two statements are equivalent:

(i) There exists $\theta \in \operatorname{Aut}\left(\mathscr{A} \bar{\otimes} \mathscr{R}_{0,1}\right)$ and $u \in Z_{\beta}^{1}\left(G \times \mathbf{R}, \mathscr{U}\left(\mathscr{A} \otimes \mathscr{R}_{0,1}\right)\right)$ such that

$$
\theta \circ \alpha_{h} \circ \theta^{-1}=\operatorname{Ad} u_{h} \circ \beta_{h}, \quad h \in G \times \mathbf{R} ;
$$

(ii) There exists $\theta \in \operatorname{Aut}(\mathscr{A})$ such that

$$
\begin{aligned}
\theta \circ \alpha_{h} \circ \theta^{-1} & =\beta_{h} \quad \text { on } \mathscr{A}, \quad h \in G \times \mathbf{R} ; \\
\theta\left(\chi_{\alpha}\right) & =\chi_{\beta} .
\end{aligned}
$$

The implication: (i) $\Rightarrow$ (ii) is routine, so we leave the details to the reader. The rest of this subsection is devoted to the proof of the implication (ii) $\Rightarrow$ (i). To this end, we need a further reduction of the problem.

We first consider $\alpha$ alone. Let $H=G \times \mathbf{R}$, and let $\alpha^{\prime}: H \ltimes X \rightarrow$ $\operatorname{Aut}\left(\mathscr{R}_{0,1}\right)$ be a point realization of this action as provided by Proposition 1.2 , and let $\bar{\alpha}$ be the restriction of $\alpha^{\prime}$ to $\mathscr{G}=\left.H \ltimes X\right|_{B}$, where $B$ is a base for the R-action on $X$. We wish to calculate the invariants as defined in [15] for the action $\bar{\alpha}$ of the discrete amenable groupoid $\mathscr{G}$ on $\mathscr{R}_{0,1}$.

To this end, define, for $x \in X$,

$$
\begin{aligned}
& \mathscr{H}_{x}=\{h \in H: h x=x\} \\
& \mathscr{N}_{x}=\left\{h \in \mathscr{H}_{x}: \alpha_{(h, x)}^{\prime} \in \operatorname{Int}\left(\mathscr{R}_{0,1}\right)\right\} .
\end{aligned}
$$

Since $\mathbf{R}$ is central in $H$, we have

$$
\begin{aligned}
& \mathscr{H}_{T_{s} x}=\mathscr{H}_{x}, \quad \text { and } \\
& \mathscr{N}_{T_{s} x}=\mathscr{N}_{x}, \quad s \in \mathbf{R} .
\end{aligned}
$$

The ergodicity of $\mathbf{R}$ then implies that we may delete a saturated null set from $X$ so that $\mathscr{H}_{x}$ and $\mathscr{N}_{x}$ are independent of $x$. Hence we may assume that $\mathscr{N}_{x}=N$ for all $x \in X$ where $N$ is a normal subgroup of 
$G$, and therefore that the "inner part" $\mathscr{N}$ of the action $\bar{\alpha}$ is nothing but $\mathscr{N}=N \times B$.

To get the invariants for $\bar{\alpha}$, choose, for each $(n, b) \in N \times B, \bar{u}(n, b) \in$ $\mathscr{U}\left(\mathscr{R}_{0,1}\right)$ such that

$$
\bar{\alpha}_{(n, b)}=\operatorname{Ad}(\bar{u}(n, b)) .
$$

Since we may choose $(n, b) \in N \times B \rightarrow \bar{u}(n, b) \in \mathscr{U}\left(\mathscr{R}_{0,1}\right)$ Borel, we obtain $\left(\lambda_{\bar{\alpha}}, \mu_{\bar{\alpha}}\right) \in Z_{\bar{\alpha}}(\mathscr{G}, \mathcal{N}, \mathbf{T})$ by defining:

$$
\begin{gathered}
\mu_{\bar{\alpha}}((m, b),(n, b))=\bar{u}(m, b) \bar{u}(n, b) \bar{u}(m n, b)^{*} ; \\
\lambda_{\bar{\alpha}}((n, g b),(g, b))=\bar{\alpha}_{(g, b)}\left(\bar{u}\left(g^{-1} n g, b\right)\right) \bar{u}(n, g b)^{*}
\end{gathered}
$$

for $(m, b),(n, b) \in \mathscr{N}$ and $((n, g b),(g, b)) \in(\mathscr{N} \times \mathscr{G}) \cap \mathscr{G}^{(2)}$.

LEMMA 4.3. $L^{\infty}\left(p^{*}\left(\lambda_{\bar{\alpha}}, \mu_{\bar{\alpha}}\right)\right)$ is cohomologous in $Z_{\alpha}(H, N, \mathscr{U}(\mathscr{A}))$ to $\left(\lambda_{\alpha}, \mu_{\alpha}\right)$.

Proof. Let $h(x) \in \mathbf{R}, p$, and $\pi$ be as in $\S 1.3$, and define

$$
u^{\prime}(n, x)=\alpha_{(h(x), \pi(x))}^{\prime}(\bar{u}(p(n, x))), \quad(n, x) \in N \times X .
$$

Then $\alpha_{(n, x)}^{\prime}=\operatorname{Ad} u^{\prime}(n, x)$ for $(n, x) \in N \times X$, so that

$$
u(n)=\int_{X}^{\oplus} u(n, x) d m^{\prime}(x)
$$

implements $\alpha_{n}$. However, we have

$$
\begin{aligned}
& u^{\prime}(m, x) u^{\prime}(n, x)=\mu_{\bar{\alpha}}((m, \pi(x)),(n, \pi(x))) u^{\prime}(m n, x) \\
& \alpha_{(g, x)}^{\prime}\left(u^{\prime}\left(g^{-1} n g, x\right)\right)=\lambda_{\bar{\alpha}}(p(n, g x), p(g, x)) u^{\prime}(n, g x)
\end{aligned}
$$

for any $g \in H$ and $x \in X$.

To determine the modular invariant for $\bar{\alpha}$, choose a Borel field $b \in$ $B \rightarrow \operatorname{Tr}_{b}$ of faithful semi-finite normal traces on $\mathscr{R}_{0,1}$, and define $\delta_{\bar{\alpha}} \in \operatorname{Hom}\left(\mathscr{G}, \mathbf{R}_{+}^{\times}\right)$by

$$
\delta_{\bar{\alpha}}(g, b) \operatorname{Tr}_{b}=\operatorname{Tr}_{g b} \circ \bar{\alpha}_{(g, b)}, \quad(g, b) \in \mathscr{G} .
$$

Define also, for each $x \in X$,

$$
\operatorname{Tr}_{x}=\operatorname{Tr}_{\pi(x)} \circ \alpha_{(h(x), \pi(x))}^{\prime-1}
$$

We then choose a Borel function: $x \in X \rightarrow k(x) \in \mathbf{R}_{+}^{\times}$such that

$$
\operatorname{Tr}=\int_{X}^{\oplus} \operatorname{Tr}_{x} k(x) d m^{\prime}(x),
$$


where $\operatorname{Tr}$ is the trace on $\mathscr{A} \otimes \mathscr{R}_{0,1}$ given in Assumption 4.1. It is easy to check that

$$
\operatorname{Tr}_{g x} \circ \alpha_{(g, x)}^{\prime}=\delta_{\bar{\alpha}}(p(g, x)) \operatorname{Tr}_{x} \quad \text { for } g \in H .
$$

If we define $\psi: G \times \mathbf{R} \rightarrow \mathbf{R}_{+}^{\times}$by

$$
\psi(g, s)=e^{-s}, \quad g \in G, s \in \mathbf{R},
$$

the fact that $\operatorname{Tr} \circ \alpha_{g}=\psi(g) \operatorname{Tr}$ for $g \in H$ may be rewritten as

$$
\begin{aligned}
\psi(g) & \int_{X}^{\oplus} k(x) \operatorname{Tr}_{x} d m^{\prime}(x)=\operatorname{Tr} \circ \alpha_{g} \\
= & \int_{X}^{\oplus} k(g x) \operatorname{Tr}_{g x} \circ \alpha_{(g, x)}^{\prime} d m^{\prime}(g x) \\
= & \int_{X}^{\oplus} k(g x) \delta_{\bar{\alpha}}(p(g, x)) k(x)^{-1} \rho^{\prime}(g, x) k(x) \operatorname{Tr}_{x} d m^{\prime}(x),
\end{aligned}
$$

where $\rho^{\prime}(g, x)=\left(d m^{\prime} \circ g / d m^{\prime}\right)(x)$ is the Radon-Nikodym cocycle of $G \times \mathbf{R}$ on $\left(X, m^{\prime}\right)$. Hence we get

$$
\psi(g)=k(g x) \delta_{\bar{\alpha}}(p(g, x)) k(x)^{-1} \rho^{\prime}(g, x), \quad g \in H, x \in X,
$$

so that we conclude that $p^{*}\left(\delta_{\bar{\alpha}}\right)$ is cohomologous in $Z^{1}\left(\mathscr{G}^{\prime}, \mathbf{R}_{+}^{\times}\right)$to the cocycle: $(g, x) \in \mathscr{G}^{\prime} \rightarrow \psi(g) \rho^{\prime}(g, x)^{-1}$.

We are now ready to prove the theorem.

Proof of Theorem 4.2. Suppose that two actions $\alpha$ and $\beta$ of $G \times \mathbf{R}$ on $\mathscr{A} \bar{\otimes} \mathscr{R}_{0,1}$ satisfy the condition (ii) of the theorem. After identifying $\alpha$ and $\theta \alpha \theta^{-1}$, we may assume that $\alpha$ and $\beta$ agree on $\mathscr{A}$ and $\chi_{\alpha}=\chi_{\beta}$. Thus we obtain point realizations $\alpha^{\prime}$ and $\beta^{\prime}$ which are actions of the same groupoid $\mathscr{G}^{\prime}=H \ltimes X$ on $\mathscr{R}_{0,1}$. If $\bar{\alpha}$ and $\bar{\beta}$ are the restrictions of $\alpha^{\prime}$ and $\beta^{\prime}$ to $\mathscr{G}=\left.\mathscr{G}^{\prime}\right|_{B}$, then the hypotheses now imply that $\chi_{\bar{\alpha}}=\chi_{\bar{\beta}}$ by Theorem 1.3 , and $\delta_{\bar{\alpha}}=\delta_{\bar{\beta}}$ in $H^{1}\left(\mathscr{G}, \mathbf{R}_{+}\right)$as seen above.

By the main result of [15], there are Borel maps $\bar{\theta}: B \rightarrow \operatorname{Aut}\left(\mathscr{R}_{0,1}\right)$ and $\bar{v} \in Z_{\bar{\alpha}}^{1}\left(\mathscr{G}, \mathscr{U}\left(\mathscr{R}_{0,1}\right)\right)$ such that

$$
\bar{\theta}_{g b} \circ \bar{\alpha}_{(g, b)} \circ \bar{\theta}_{b}^{-1}=\operatorname{Ad}(\bar{v}(g, b)) \circ \bar{\beta}_{(g, b)}, \quad(g, b) \in \mathscr{G} .
$$

Define $\theta^{\prime}: X \rightarrow \operatorname{Aut}\left(\mathscr{R}_{0,1}\right)$ and $v^{\prime}: \mathscr{G}^{\prime} \rightarrow \mathscr{U}\left(\mathscr{R}_{0,1}\right)$ by

$$
\begin{aligned}
\theta_{x}^{\prime} & =\bar{\beta}_{(h(x), \pi(x))} \circ \bar{\theta}_{\pi(x)} \circ \bar{\alpha}_{(h(x), \pi(x))}^{-1} ; \\
v^{\prime}(g, x) & =\beta_{(h(g x), \pi(g x))}(\bar{v}(p(g, x))) .
\end{aligned}
$$

Evidently, $\theta^{\prime}$ and $v^{\prime}$ are both Borel maps; it is routine to check that $v^{\prime} \in Z_{\beta^{\prime}}^{1}\left(\mathscr{G}^{\prime}, \mathscr{U}\left(\mathscr{R}_{0,1}\right)\right)$ and that

$$
\theta_{g x}^{\prime} \circ \alpha_{(g, x)}^{\prime} \circ \theta_{x}^{\prime-1}=\operatorname{Ad}\left(v^{\prime}(g, x)\right) \circ \beta_{(g, x)}^{\prime}, \quad(g, x) \in \mathscr{G}^{\prime} .
$$


Setting

$$
\begin{aligned}
\theta & =\int_{X}^{\oplus} \theta_{x}^{\prime} d m^{\prime}(x) ; \\
v(g) & =\int_{X}^{\oplus} v^{\prime}(g, x) d m^{\prime}(x), \quad g \in G \times \mathbf{R},
\end{aligned}
$$

we conclude that

$$
\theta \circ \alpha_{g} \circ \theta^{-1}=\operatorname{Ad}(v(g)) \circ \beta_{g}, v \in Z_{\beta}^{1}\left(G \times \mathbf{R}, \mathscr{U}\left(\mathscr{A} \bar{\otimes} \mathscr{R}_{0,1}\right)\right),
$$

and that $\theta$ commutes with $\left\{\alpha_{g}: g \in G \times \mathbf{R}\right\}$ on $\mathscr{A}$.

4.2 Realization of the invariants. In $\S 4.1$, we have shown that actions of $G \times \mathbf{R}$ on $\mathscr{A} \bar{\otimes}_{0,1}$ satisfying Assumption 4.1 are classified up to cocycle conjugacy in terms of the associated characteristic invariant. The following result tells us which invariants actually occur for these actions.

Theorem 4.4. Suppose that $\beta: G \times \mathbf{R} \rightarrow \operatorname{Aut}(\mathscr{A})$ is an action of $G \times \mathbf{R}$ on an abelian von Neumann algebra $\mathscr{A}$ with $\mathscr{A} \neq \mathbf{C}$, and that the restriction of $\beta$ to $\mathbf{R}$ is ergodic. Suppose further that $G$ is a countable discrete amenable group as above, and that $N$ is a normal subgroup of $G$ which acts trivially on $\mathscr{A}$. For every $(\lambda, \mu) \in Z_{\beta}(G \times \mathbf{R}, N, \mathscr{U}(\mathscr{A}))$, there exists an action $\alpha$ of $G \times \mathbf{R}$ on $\mathscr{A} \bar{\otimes} \mathscr{R}_{0,1}$ such that

(i) $\alpha$ satisfies Assumption 4.1;

(ii) $\alpha$ restricted to $\mathscr{A}$ is $\beta$;

(iii) $\chi_{\alpha}=[\lambda, \mu]$ in $\Lambda_{\beta}(G \times \mathbf{R}, N, \mathscr{U}(\mathscr{A}))$.

Proof. Let $\mathscr{G}^{\prime}=H \ltimes X, H=G \times \mathbf{R}$, be the groupoid arising from a point realization of $\left\{\beta_{g}: g \in H\right\}$ on $\mathscr{A}$, and let $B$ be a base for the Raction on $X$. Set $\mathscr{G}=\left.\mathscr{G}^{\prime}\right|_{B}$. By Theorem 1.2 , we may choose $(\bar{\lambda}, \bar{\mu}) \in$ $Z(\mathscr{G}, \mathscr{N}=N \times B, \mathbf{T})$ with $L^{\infty}\left(p^{*}(\lambda, \mu)\right)=(\bar{\lambda}, \bar{\mu})$ up to cohomology. Let $\psi$ be the homomorphism of $H$ into $\mathbf{R}_{+}^{\times}$defined by (4.13) and $\rho^{\prime} \in Z^{1}\left(\mathscr{G}, \mathbf{R}_{+}^{\times}\right)$be the Radon-Nikodym cocycle, i.e.

$$
\rho^{\prime}(g, x)=\frac{d m^{\prime} \circ g}{d m^{\prime}}(x), \quad g \in H, x \in X .
$$

Then a variant of Theorem 1.3 guarantees the existence of $\bar{\delta} \in$ $Z^{1}\left(\mathscr{G}, \mathbf{R}_{+}^{\times}\right)$such that the cocycle $(g, x) \in \mathscr{G}^{\prime} \rightarrow \psi(g) \rho^{\prime}(g, x)^{-1} \in \mathbf{R}_{+}^{\times}$is cohomologous to $\left(p^{*} \bar{\delta}\right)(g, x)$.

By the realization theorem of [15], there exists an action $\bar{\alpha}$ of $\mathscr{G}$ on $\mathscr{R}_{0,1}$ whose associated invariants are precisely

$$
\begin{aligned}
\chi_{\bar{\alpha}} & =[\bar{\lambda}, \bar{\mu}] \in \Lambda(\mathscr{G}, \mathscr{N}, \mathbf{T}), \\
{\left[\delta_{\bar{\alpha}}\right] } & =[\bar{\delta}] \in H^{1}\left(\mathscr{G}, \mathbf{R}_{+}^{\times}\right) .
\end{aligned}
$$


Define an action $\alpha^{\prime}$ of $\mathscr{G}^{\prime}$ on $\mathscr{R}_{0,1}$ by

$$
\alpha_{(g, x)}^{\prime}=\bar{\alpha}_{p(g, x)}, \quad(g, x) \in \mathscr{G}^{\prime},
$$

and set

$$
\alpha_{g}(a)(g x)=\alpha_{(g, x)}^{\prime}(a(x)), \quad a(\cdot) \in \mathscr{A} \bar{\otimes}_{0,1} .
$$

It is then clear that $\left\{G \times \mathbf{R}, \alpha, \mathscr{A} \otimes \mathscr{R}_{0,1}\right\}$ is an action which satisfies our Assumption 4.1 and for which $\chi_{\alpha}=[\lambda, \mu]$.

5. Actions on injective factors of type $\mathrm{III}_{\lambda}, \lambda \neq 1$. Throughout this section, $G$ will denote a discrete countable amenable group, and $\mathscr{M}$ an injective factor of type III $_{\lambda}, \lambda \neq 1$, acting (when necessary) on a separable Hilbert space $\mathscr{H}$. Let $\mathscr{W}(\mathscr{M})$ denote the space of semifinite normal weights on $\mathscr{M}$ and $\mathscr{W}_{d}(\mathscr{M})$ the set of dominant weights (see [4, §II.2]). The smooth flow of weights on $\mathscr{M}$ will be denoted by $\{\mathscr{F}(\mathscr{M}), F\}$, and its first unitary cocycle group by $Z^{1}(\mathscr{F}(\mathscr{M}))$. (Here we use the additive group $\mathbf{R}$ instead of the multiplicative group $\mathbf{R}_{+}^{\times}$ under the identification: $s \in \mathbf{R} \leftrightarrow e^{s} \in \mathbf{R}_{+}^{\times}$.) Each $c \in Z^{1}(\mathscr{F}(\mathscr{M}))$ gives rise to an extended modular automorphism $\bar{\sigma}_{c}^{\phi}$ for each $\phi \in \mathscr{W}_{d}(\mathscr{M})$, (see [4, §IV.2]).

For each $\phi \in \mathscr{W}_{d}(\mathscr{M})$, we define a group $\tilde{\mathscr{U}}_{\phi}(\mathscr{M})$ by

$$
\tilde{\mathscr{U}}_{\phi}(\mathscr{M})=\mathscr{U}(\mathscr{M}) \ltimes_{\phi} Z^{1}(\mathscr{F}(\mathscr{M})) \text {, }
$$

where the product is given as follows:

$$
(u, c)(v, d)=\left(u \bar{\sigma}_{c}^{\phi}(v), c d\right)
$$

By $[3, \S 3.8]$ we have an exact sequence:

$$
1 \rightarrow K_{\phi} \rightarrow \tilde{\mathscr{U}}_{\phi}(\mathscr{M}) \stackrel{\operatorname{Ad}_{\phi}}{\longrightarrow} \operatorname{Cnt}(\mathscr{M}) \rightarrow 1,
$$

where $\operatorname{Cnt}(\mathscr{M})$ denotes the group of centrally trivial automorphisms of $\mathscr{M}, \mathrm{Ad}_{\phi}$ is the map given by

$$
\operatorname{Ad}_{\phi}(u, c)=\operatorname{Ad}(u) \circ \bar{\sigma}_{c}^{\phi}, \quad(u, c) \in \tilde{\mathscr{U}}_{\phi}(\mathscr{M}),
$$

and $K_{\phi}$ is simply the kernel of $\operatorname{Ad}_{\phi}$.

LEMMA 5.1. The normal subgroup $K_{\phi}$ of $\tilde{\mathscr{U}}_{\phi}(\mathscr{M})$ is central and isomorphic to $\mathscr{U}\left(\mathscr{C}_{\phi}\right)$ under the map:

$$
u \in \mathscr{U}\left(\mathscr{C}_{\phi}\right) \rightarrow\left(u, \partial_{\phi} u\right) \in K_{\phi},
$$

where $\mathscr{C}_{\phi}$ is the centre of the centralizer $\mathscr{M}_{\phi}$ and

$$
\left(\partial_{\phi} u\right)(s)=p_{\phi}\left(u^{*}\right) F_{s}\left(p_{\phi}(u)\right) .
$$


Here $p_{\phi}$ is the isomorphism of $\mathscr{C}_{\phi}$ onto $\mathscr{F}(\mathscr{M})$ described in [4, Theorem I.11]. In particular, $K_{\phi}$ is abelian.

Proof. This assertion follows from the following easy chain of equivalences for $(u, c) \in \tilde{\mathscr{U}}_{\phi}(\mathscr{M})$ :

$$
\begin{gathered}
(u, c) \in K_{\phi} \Leftrightarrow \operatorname{Ad}(u) \circ \bar{\sigma}_{c}^{\phi}=\imath \Leftrightarrow \bar{\sigma}_{c}^{\phi}=\operatorname{Ad}\left(u^{*}\right) \Leftrightarrow \\
u^{*} \in C_{\phi} \quad \text { and } c(s)=p_{\phi}\left(u^{*}\right) F_{s}\left(p_{\phi}(u)\right) .
\end{gathered}
$$

Here the last step requires the relative commutant theorem of [4]. A direct computation shows that $K_{\phi}$ is central.

In view of the above fact, we define an isomorphism $q_{\phi}: \mathscr{U}(\mathscr{F}(\mathscr{M}))$ $\rightarrow K_{\phi}$ as follows:

$$
q_{\phi}(u)=\left(p_{\phi}^{-1}(u), \partial_{F} u\right) \in K_{\phi}, \quad u \in \mathscr{U}(\mathscr{F}(\mathscr{M})),
$$

where

$$
\left(\partial_{F} u\right)(s)=u^{*} F_{s}(u), \quad s \in \mathbf{R} .
$$

Thus we obtain an exact sequence:

$$
1 \rightarrow \mathscr{U}(\mathscr{F}(\mathscr{M})) \stackrel{q_{\phi}}{\longrightarrow} \tilde{\mathscr{U}}_{\phi}(\mathscr{M}) \stackrel{\operatorname{Ad}_{\phi}}{\longrightarrow} \operatorname{Cnt}(\mathscr{M}) \rightarrow 1 .
$$

We will need to view this exact sequence in the category of Aut $(\mathscr{M})$ "modules". The action of $\operatorname{Aut}(\mathscr{M})$ on $\operatorname{Cnt}(\mathscr{M})$ will be by conjugation, $\beta \in \operatorname{Cnt}(\mathscr{M}) \rightarrow \alpha \beta \alpha^{-1} \in \operatorname{Cnt}(\mathscr{M})$ for $\alpha \in \operatorname{Aut}(\mathscr{M})$; the action on $\mathscr{U}(\mathscr{F}(\mathscr{M}))$ will be the module action $\bmod$ of $[4$, p. 549]. For $\alpha \in$ Aut $(\mathscr{M})$ and $(u, c) \in \tilde{\mathscr{U}}_{\phi}(\mathscr{M})$, we define

$$
\tilde{\alpha}_{\phi}(u, c)=\left(\alpha(u)\left(D \phi \circ \alpha^{-1}: D \phi\right)(\bmod \alpha)(c),(\bmod \alpha)(c)\right) .
$$

In order to take the action of $\operatorname{Aut}(\mathscr{M})$ on the exact sequence $\left(5.3^{\prime}\right)$ into account, we need the next easy result:

LEMMA 5.2. If $\phi, \psi \in \mathscr{W}_{d}(\mathscr{M})$ and $v \in \mathscr{U}(\mathscr{F}(\mathscr{M}))$, we have

$$
\begin{aligned}
\bar{\sigma}_{\partial_{F} v}^{\phi} & =\operatorname{Ad} p_{\phi}^{-1}\left(v^{*}\right), \\
(D \psi: D \phi)_{\partial_{F} v} & =p_{\psi}^{-1}\left(v^{*}\right) p_{\phi}^{-1}(v) .
\end{aligned}
$$

Proof. In the continuous decomposition, $\mathscr{M}=\mathscr{M}_{\phi} \ltimes_{\theta} \mathbf{R}$, of $\mathscr{M}$, ([4, 15]) let $\{u(s)\}$ be the one parameter unitary group corresponding to $\theta$. Then we have for each $c \in Z_{F}^{1}(\mathbf{R}, \mathscr{U}(\mathscr{F}(\mathscr{M})))$,

$$
\bar{\sigma}_{c}^{\phi}(a u(s))=p_{\phi}^{-1}(c(s)) a u(s), \quad a \in \mathscr{M}_{\phi}, s \in \mathbf{R},
$$


so that

$$
\bar{\sigma}_{\partial_{F} v}^{\phi}(a u(s))=p_{\phi}^{-1}\left(v^{*} F_{s}(v)\right) a u(s)=p_{\phi}^{-1}\left(v^{*}\right) a u(s) p_{\phi}^{-1}(v) .
$$

Hence (5.8) follows.

For (5.9), consider the balanced weight $\rho=\phi \oplus \psi$ on $M_{2}(\mathscr{M})$ and compute:

$(D \psi: D \phi)_{\partial_{F} v} \otimes e_{21}=\bar{\sigma}_{\partial_{F} v}^{\rho}\left(1 \otimes e_{21}\right)=p_{\rho}^{-1}\left(v^{*}\right)\left(1 \otimes e_{21}\right) p_{\rho}^{-1}(v)$.

Since $p_{\rho}^{-1}\left(v^{*}\right)=p_{\phi}^{-1}\left(v^{*}\right) \otimes e_{11}+p_{\psi}^{-1}\left(v^{*}\right) \otimes e_{22}$, the formula (5.9) follows.

We now have the following:

LEMMA 5.3. The exact sequence $\left(5.3^{\prime}\right)$ is an exact sequence of $\operatorname{Aut}(\mathscr{M})$-modules.

Proof. We first show that the map: $\alpha \in \operatorname{Aut}(\mathscr{M}) \rightarrow \tilde{\alpha}_{\phi}$ is actually a homomorphism of $\operatorname{Aut}(\mathscr{M})$ into $\operatorname{Aut}\left(\tilde{\mathscr{U}}_{\phi}(\mathscr{M})\right)$. For this, we calculate, using [4, §IV.2] or [13, p. 405], as follows: for each $\alpha \in \operatorname{Aut}(\mathscr{M})$ and $(u, c),(v, d) \in \tilde{\mathscr{U}}_{\phi}(\mathscr{M})$,

$$
\begin{aligned}
\tilde{\alpha}_{\phi}\left(u \bar{\sigma}_{c}^{\phi}\right. & (v), c d) \\
= & \left(\alpha\left(u \bar{\sigma}_{c}^{\phi}(v)\right)\left(D \phi \circ \alpha^{-1}: D \phi\right)_{(\bmod \alpha)(c d),(\bmod \alpha)(c d))}\right. \\
= & \left(\alpha(u) \bar{\sigma}_{(\bmod \alpha)(c)}^{\phi \circ \alpha^{-1}}(\alpha(v))\left(D \phi \circ \alpha^{-1}: D \phi\right)(\bmod \alpha)(c)\right. \\
& \left.\times \bar{\sigma}_{(\bmod \alpha)(c)}^{\phi}\left(\left(D \phi \circ \alpha^{-1}: D \phi\right)(\bmod \alpha)(d)\right),(\bmod \alpha)(c d)\right) \\
= & \left(\alpha(u)\left(D \phi \circ \alpha^{-1}: D \phi\right)(\bmod \alpha)(c)\right. \\
& \left.\times \bar{\sigma}_{(\bmod \alpha)(c)}^{\phi}\left(\alpha(v)\left(D \phi \circ \alpha^{-1}: D \phi\right)(\bmod \alpha)(d)\right),(\bmod \alpha)(c d)\right) \\
= & \tilde{\alpha}_{\phi}(u, c) \tilde{\alpha}_{\phi}(v, d) .
\end{aligned}
$$

So $\tilde{\alpha}_{\phi}$ is an automorphism of $\tilde{\mathscr{U}}_{\phi}(\mathscr{M})$. Similarly, for $\alpha, \beta \in \operatorname{Aut}(\mathscr{M})$ and $(u, c) \in \tilde{\mathscr{U}}_{\phi}(\mathscr{M})$, we have

$$
\begin{aligned}
\tilde{\alpha}_{\phi} \tilde{\beta}_{\phi}(u, c)= & \tilde{\alpha}_{\phi}\left(\beta(u)\left(D \phi \circ \beta^{-1}: D \phi\right)(\bmod \beta)(c),(\bmod \beta)(c)\right) \\
= & \left(\alpha \beta(u) \alpha\left(\left(D \phi \circ \beta^{-1}: D \phi\right)(\bmod \beta)(c)\right)\right. \\
& \left.\times\left(D \phi \circ \alpha^{-1}: D \phi\right)(\bmod \alpha \beta)(c),(\bmod \alpha \beta)(c)\right) \\
= & \left(\alpha \beta(u)\left(D \phi \circ \beta^{-1} \circ \alpha^{-1}: D \phi \circ \alpha^{-1}\right)(\bmod \alpha \beta)(c)\right. \\
& \left.\times\left(D \phi \circ \alpha^{-1}: D \phi\right)(\bmod \alpha \beta)(c),(\bmod \alpha \beta)(c)\right) \\
= & \left(\alpha \beta(u)\left(D \phi \circ \beta^{-1} \circ \alpha^{-1}: D \phi\right)(\bmod \alpha \beta)(c),(\bmod \alpha \beta)(c)\right) \\
= & (\alpha \beta)_{\phi}(u, c),
\end{aligned}
$$

so that $\alpha \rightarrow \tilde{\alpha}_{\phi}$ is a homomorphism. 
We next prove that $\operatorname{Ad}_{\phi} \tilde{\alpha}_{\phi}(u, c)=\alpha \circ \operatorname{Ad}_{\phi}(u, c) \circ \alpha^{-1}$. This is seen by the following calculation:

$$
\begin{aligned}
& \operatorname{Ad}\left(\alpha(u)\left(D \phi \circ \alpha^{-1}: D \phi\right)_{(\bmod \alpha)(c)}\right) \circ \bar{\sigma}_{(\bmod \alpha)(c)}^{\phi} \\
& \quad=\operatorname{Ad}(\alpha(u)) \circ \bar{\sigma}_{(\bmod \alpha)(c)}^{\phi \circ \alpha^{-1}}=\alpha \operatorname{Ad}(u) \circ \bar{\sigma}_{c}^{\phi} \circ \alpha^{-1} .
\end{aligned}
$$

It now remains to show that for $u \in \mathscr{U}(\mathscr{F}(\mathscr{M}))$

$$
q_{\phi}(\bmod \alpha(u))=\tilde{\alpha}_{\phi}\left(q_{\phi}(u)\right) .
$$

Now, we have

whilst

$$
q_{\phi}(\bmod \alpha(u))=\left(p_{\phi}^{-1}(\bmod \alpha(u)), \partial_{F}(\bmod \alpha(u)),\right.
$$

$$
\begin{aligned}
\tilde{\alpha}\left(q_{\phi}(u)\right) & =\tilde{\alpha}_{\phi}\left(p_{\phi}^{-1}(u), \partial_{F} u\right) \\
& =\left(\alpha\left(p_{\phi}^{-1}(u)\right)\left(D \phi \circ \alpha^{-1}: D \phi\right)(\bmod \alpha)\left(\partial_{F} u\right), \bmod \alpha\left(\partial_{F} u\right)\right) .
\end{aligned}
$$

Since $\partial_{F}(\bmod \alpha(u))=\bmod \alpha\left(\partial_{F} u\right)$, it suffices to show that

$$
\left.\alpha\left(p_{\phi}^{-1}(u)\right)\left(D \phi \circ \alpha^{-1}: D \phi\right)(\bmod \alpha)\left(\partial_{F} u\right)=p_{\phi}^{-1} \bmod \alpha(u)\right) .
$$

By Lemma 5.2, we have

$$
\begin{gathered}
\left(D \phi \circ \alpha^{-1}: D \phi\right)(\bmod \alpha)\left(\partial_{F} u\right)=\left(D \phi \circ \alpha^{-1}: D \phi\right)_{\partial_{F}(\bmod \alpha(u))} \\
=p_{\phi \circ \alpha^{-1}}^{-1}\left(\bmod \alpha\left(u^{*}\right)\right) p_{\phi}^{-1}(\bmod \alpha(u)),
\end{gathered}
$$

so that the fact $\alpha \circ p_{\phi}=p_{\phi \circ \alpha^{-1}}^{-1} \circ \bmod \alpha$, yields the desired conclusion.

In order to remove the dependence of the exact sequence $\left(5.3^{\prime}\right)$ on the choice of $\phi \in \mathscr{W}_{d}(\mathscr{M})$, we introduce the maps $\kappa_{\psi, \phi}: \tilde{\mathscr{U}}_{\phi}(\mathscr{M}) \rightarrow$ $\tilde{\mathscr{U}}_{\psi}(\mathscr{M})$ given by

$$
\kappa_{\psi, \phi}(u, c)=\left(u(D \phi: D \psi)_{c}, c\right)
$$

and prove:

LEMMA 5.4. (i) The map $\kappa_{\phi, \psi}$ is an isomorphism.

(ii) The following diagram commutes and is Aut( $\mathscr{M})$-equivariant:

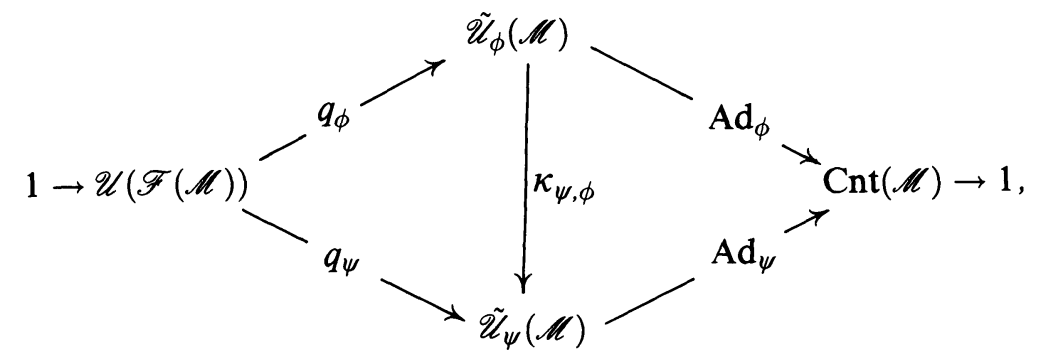

for $\phi, \psi \in \mathscr{W}_{d}(\mathscr{M})$. 
(iii) For $\phi$, $\psi$ and $\rho \in \mathscr{W}_{d}(\mathscr{M})$, we have

$$
\kappa_{\phi, \psi} \kappa_{\psi, \phi}=\kappa_{\phi, \rho}
$$

Proof. The facts that $\kappa_{\psi, \phi}$ is an $\operatorname{Aut}(\mathscr{M})$-equivariant isomorphism, and that $\operatorname{Ad}_{\psi} \circ \kappa_{\psi, \phi}=\operatorname{Ad}_{\phi}$ both follow from routine calculations using [4, §IV.2] or [13, p. 405].

For each $u \in \mathscr{U}(\mathscr{F}(\mathscr{M}))$, we have, by Lemma 5.2,

$$
\begin{aligned}
\kappa_{\psi, \phi} & \circ q_{\phi}(u)=\left(p_{\phi}^{-1}(u)(D \psi: D \phi)_{\partial_{F} u}, \partial_{F} u\right) \\
& =\left(p_{\phi}^{-1}(u) p_{\phi}^{-1}\left(u^{*}\right) p_{\psi}^{-1}(u), \partial_{F} u\right)=\left(p_{\psi}^{-1}(u), \partial_{F} u\right)=q_{\psi}(u)
\end{aligned}
$$

The last assertion (iii) is a trivial consequence of the chain rule for the Radon-Nikodym cocycle derivatives.

The groups $\tilde{\mathscr{U}}_{\phi}(\mathscr{M})$, and the exact sequences of $\left(5.3^{\prime}\right)$ are thus independent, up to isomorphism, of the choice of weight $\phi \in \mathscr{W}_{d}(\mathscr{M})$. At this point, we know of no intrinsic description of this group, and so are obliged to remove the dependence of $\phi$ by the following device:

Definition 5.5. We set

(5.15) $\tilde{\mathscr{U}}(\mathscr{M})=\left\{\left(\tilde{u}_{\phi}\right)_{\phi} \in \prod_{\phi \in \mathscr{W}_{d}} \tilde{\mathscr{U}}_{\phi}(\mathscr{M}): \kappa_{\phi, \psi}\left(\tilde{u}_{\psi}\right)=\tilde{u}_{\phi}, \phi, \psi \in \mathscr{W}_{d}\right\}$.

Evidently, we have now an $\operatorname{Aut}(\mathscr{M})$ equivariant short exact sequence:

$$
1 \rightarrow \mathscr{U}(\mathscr{F}(\mathscr{M})) \stackrel{q}{\rightarrow} \tilde{\mathscr{U}}(\mathscr{M}) \stackrel{\text { Ad }}{\longrightarrow} \operatorname{Cnt}(\mathscr{M}) \rightarrow 1,
$$

where we set, using Lemma 5.4,

$$
\begin{gathered}
q(u)_{\phi}=q_{\phi}(u), \quad u \in \mathscr{U}(\mathscr{F}(\mathscr{M})) ; \\
\operatorname{Ad}\left(\left(u_{\phi}\right)\right)=\operatorname{Ad}_{\phi}\left(u_{\phi}\right), \quad\left(u_{\phi}\right) \in \tilde{\mathscr{U}}(\mathscr{M}) .
\end{gathered}
$$

Before using the exact sequence (5.16) to construct invariants for actions, we need one further preparatory result. We omit, however, the proof, as all computations involved are routine.

LEMMA 5.6. For $w \in \mathscr{U}(\mathscr{M})$, set

$$
\tilde{w}=\left((w, 1)_{\phi}\right) \in \tilde{\mathscr{U}}(\mathscr{M}) .
$$


Let $\tilde{v} \in \tilde{\mathscr{U}}(\mathscr{M})$ be arbitrary. Then

(i) The map $w \in \mathscr{U}(\mathscr{M}) \rightarrow \tilde{w} \in \tilde{\mathscr{U}}(\mathscr{M})$ is an $\operatorname{Aut}(\mathscr{M})$ - equivariant homomorphism;

(ii) $\tilde{v} \tilde{w}(\tilde{v})^{-1}=[\operatorname{Ad}(\tilde{v})(w)]^{\sim}$;

(iii) $\operatorname{Ad} \tilde{w}=\operatorname{Ad} w$.

Now, let $\alpha$ be an action of $G$ on $\mathscr{M}$. The exact sequence (5.13) may then be viewed as an exact sequence of $G$-modules. The restriction of $\alpha$ to

$$
N(\alpha)=\alpha^{-1}(\operatorname{Cnt}(\mathscr{M}))
$$

now canonically defines an element

$$
\chi_{\alpha}=\left[\lambda_{\alpha}, \mu_{\alpha}\right] \in \Lambda(G, N(\alpha), \mathscr{U}(\mathscr{F}(\mathscr{M})))
$$

as in [11, p. 8]. More specifically, for each $n \in N(\alpha)$ choose $\tilde{u}(n) \in$ $\tilde{\mathscr{U}}(\mathscr{M})$ with $\alpha_{n}=\operatorname{Ad}(\tilde{u}(n))$, and note that we have

$$
\begin{gathered}
\tilde{u}(m) \tilde{u}(n)=\mu_{\alpha}(m, n) \tilde{u}(m n), \quad m, n \in N(\alpha), \\
\tilde{\alpha}_{g}\left(\tilde{u}\left(g^{-1} n g\right)\right)=\lambda_{\alpha}(n, g) \tilde{u}(n), \quad g \in G,
\end{gathered}
$$

where $\mu_{\alpha}: N(\alpha) \times N(\alpha) \rightarrow \mathscr{U}(\mathscr{F}(\mathscr{M}))$ and $\lambda_{\alpha}: N(\alpha) \times G \rightarrow \mathscr{U}(\mathscr{F}(\mathscr{M}))$. The pair $\left(\lambda_{\alpha}, \mu_{\alpha}\right)$ is an element of $Z(G, N(\alpha), \mathscr{U}(\mathscr{F}(\mathscr{M})))$, and hence defines an element $\chi_{\alpha}$ of $\Lambda(G, N(\alpha), \mathscr{U}(\mathscr{F}(\mathscr{M})))$.

There is however another invariant coming from the obvious homomorphism of $\tilde{\mathscr{U}}(\mathscr{M})$ onto $Z^{1}(\mathscr{F}(\mathscr{M}))$, the projection to the second component in (5.1), which induces a homomorphism of $\tilde{\mathscr{U}}(\mathscr{M})$ onto $H^{1}(\mathscr{F}(\mathscr{M}))$. With $\tilde{u}(n), n \in N(\alpha)$, as above, we write

$$
\tilde{u}(n)=\left(u_{\phi}(n), c(n)\right)_{\phi \in \mathscr{W}_{d}} \in \tilde{\mathscr{U}}(\mathscr{M}),
$$

and set, for each $n \in N(\alpha)$,

$$
\nu_{\alpha}(n)=[c(n)] \in H^{1}(\mathscr{F}(\mathscr{M})) .
$$

The previous arguments show that the cohomology class $\nu_{\alpha}(n)$ is independent of the choice of $\{u(n)\}$, so that $\nu_{\alpha}$ is an invariant of $\alpha$.

Proposition 5.7. Let $\alpha$ and $\beta$ be two actions of $G$ on $\mathscr{M}$. Suppose that

$$
\theta \circ \alpha_{g} \circ \theta^{-1}=\operatorname{Ad}(w(g)) \circ \beta_{g}, \quad g \in G,
$$

with $\theta \in \operatorname{Aut}(\mathscr{M})$ and $w \in Z_{\beta}^{1}(G, \mathscr{U}(\mathscr{M}))$. Then $N(\alpha)=N(\beta)$ and

$$
\theta\left(\bmod \alpha, \chi_{\alpha}, \nu_{\alpha}\right)=\left(\bmod \beta, \chi_{\beta}, \nu_{\beta}\right) \text {, }
$$


where $\operatorname{Aut}(\mathscr{M})$ acts on

$$
\operatorname{Aut}(\mathscr{F}(\mathscr{M})) \times \Lambda(G, N(\alpha), \mathscr{U}(\mathscr{F}(\mathscr{M}))) \times \operatorname{Hom}\left(N, H^{1}(\mathscr{F}(\mathscr{M}))\right)
$$

via the diagonal action coming from the module.

Proof. It is trivial that $N(\alpha)=N(\beta)$, since $\operatorname{Int}(\mathscr{M}) \subseteq \operatorname{Cnt}(\mathscr{M})$ and $\operatorname{Cnt}(\mathscr{M})$ is normal in $\operatorname{Aut}(\mathscr{M})$.

Similarly, since $\operatorname{Int}(\mathscr{M}) \subseteq \operatorname{ker}(\bmod )$,

$$
\theta(\bmod \alpha)=\bmod (\theta) \bmod (\alpha)(\bmod \theta)^{-1}=\bmod \left(\theta \alpha \theta^{-1}\right)=\bmod \beta .
$$

To prove the remaining assertions, choose $\tilde{v}(n) \in \tilde{\mathscr{U}}(\mathscr{M})$ for $n \in$ $N(\alpha)=N(\beta)$, say $N$, with Ad $\tilde{v}(n)=\beta_{n}$. By Lemma 5.6, we have

$$
\operatorname{Ad} w(n) \circ \beta_{n}=\operatorname{Ad}\left(w(n)^{\sim} \tilde{v}(n)\right) ;
$$

however, for $m, n \in N$, we have

$$
\begin{gathered}
w(m)^{\sim} \tilde{v}(m) w(n)^{\sim} \tilde{v}(n)=w(m)^{\sim} \beta_{m}(w(n))^{\sim} \tilde{v}(m) \tilde{v}(n) \\
=w(m n)^{\sim} \tilde{v}(m) \tilde{v}(n)=\mu_{\beta}(m, n) w(m n)^{\sim} \tilde{v}(m n),
\end{gathered}
$$

using $w \in Z_{\beta}^{1}(G, \mathscr{U}(\mathscr{M}))$, Lemma 5.6 and the centrality of $\mathscr{U}(\mathscr{F}(\mathscr{M}))$ in $\tilde{\mathscr{U}}(\mathscr{M})$.

Similarly, for $n \in N$ and $g \in G$, we have

$$
\begin{aligned}
(\operatorname{Ad} & \left.w(g) \circ \beta_{g}\right)^{\sim}\left(w\left(g^{-1} n g\right)^{\sim} \tilde{v}\left(g^{-1} n g\right)\right) \\
& =w(g)^{\sim} \beta_{g}\left(w\left(g^{-1} n g\right)\right)^{\sim} \tilde{\beta}_{g}\left(\tilde{v}\left(g^{-1} n g\right)\right)\left(w(g)^{*}\right)^{\sim} \\
& =\left(w(g) \beta_{g}\left(w\left(g^{-1}\right)\right) w(n) \beta_{n}(w(g))\right)^{\sim} \lambda_{\beta}(n, g) \tilde{v}(n)\left(w(g)^{\sim}\right)^{-1} \\
& =w(n)^{\sim} \beta_{n}\left(w(g) w(g)^{-1}\right)^{\sim} \lambda_{\beta}(n, g) \tilde{v}(n) \\
& =\lambda_{\beta}(n, g) w(n)^{\sim} \tilde{v}(n) .
\end{aligned}
$$

These calculations show that there are choices of implementing elements in $\tilde{\mathscr{U}}(\mathscr{M})$ for $\beta_{n}$ and $\operatorname{Ad}(w(n)) \circ \beta_{n}, n \in N$, for which the corresponding elements in $Z_{\beta}(G, N, \mathscr{U}(\mathscr{F}(\mathscr{M})))$ agree. The assertions that $\theta\left(\nu_{\alpha}\right)=\nu_{\beta}$ and $\theta\left(\chi_{\alpha}\right)=\chi_{\beta}$ follow now immediately.

Definition 5.8. The invariant $\nu_{\alpha}$ of $\alpha$ in the previous proposition will be called the modular invariant of $\alpha$. We caution that the modular invariant $\nu_{\alpha}$ of $\alpha$ should not be confused with the module $\bmod \alpha$ of $\alpha$. They are in some sense dual to one another.

We now turn to our main result on classification of actions. 
THEOREM 5.9. Let $\mathscr{M}$ be an injective factor of type $\mathrm{III}_{\lambda}, \lambda \neq 1$, and let $\alpha, \beta$ be actions of a countable amenable group $G$ on $\mathscr{M}$. Then $\alpha$ and $\beta$ are cocycle conjugate if and only if

(i) $N(\alpha)=N(\beta)$;

(ii) For some $\theta \in \operatorname{Aut}(\mathscr{F}(\mathscr{M}))$, we have

$$
\theta\left(\bmod \alpha, \chi_{\alpha}, \nu_{\alpha}\right)=\left(\bmod \beta, \chi_{\beta}, \nu_{\beta}\right) \text {. }
$$

One implication has been established in Proposition 5.7. The converse will be shown via the following sequence of lemmas.

LEMMA 5.10. If $\alpha$ is an action of $G$ on $\mathscr{M}$, then $\alpha$ is cocycle conjugate to an action $\alpha^{\prime}$ which admits an invariant dominant weight.

Proof. Let $\phi$ be an arbitrary faithful semi-finite normal weight on $\mathscr{M}$, and let $\hat{\hat{\phi}}$ be the second dual weight on $\left(\mathscr{M} \ltimes_{\alpha} G\right) \ltimes_{\hat{\alpha}} G=\mathscr{M} \bar{\otimes} \mathscr{L}\left(l^{2}(G)\right)$. By [10], $\hat{\hat{\phi}}$ is invariant under the second dual action $\hat{\hat{\alpha}}$. If $\omega$ is the weight on the $I_{\infty}$ factor $\mathscr{L}\left(L^{2}(\mathbf{R})\right)$ described in [4, p. 496], then $\hat{\hat{\phi}} \otimes \omega$ is dominant and invariant under $\hat{\hat{\alpha}} \otimes \mathrm{id}$. However, by [16, Lemma 4.7], $\alpha$ is cocycle conjugate to $\hat{\hat{\alpha}} \otimes \mathrm{id}$.

LEMMA 5.11. If $\alpha$ is an action of $G$ on $\mathscr{M}$, then there is an action $\alpha^{\prime}$ of $G$ on $\mathscr{M}$ and a dominant weight $\phi$ on $\mathscr{M}$ such that

(i) $\alpha^{\prime}$ is cocycle conjugate to $\alpha$;

(ii) $\phi$ is $\alpha^{\prime}$-invariant;

(iii) There is a one parameter unitary group $\{u(s): s \in \mathbf{R}\}$ such that

$$
\begin{aligned}
& \sigma_{t}^{\phi}(u(s))=e^{i s t} u(s), \quad s, t \in \mathbf{R} ; \\
& \alpha_{g}(u(s))=u(s), \quad g \in G .
\end{aligned}
$$

Proof. By the last lemma, we may assume that $\alpha$ admits an invariant dominant weight $\psi$ on $\mathscr{M}$. We consider the action $\alpha_{g}^{\prime}=\alpha_{g} \otimes \rho_{g}$ and the weight $\phi=\psi \otimes \operatorname{Tr}$ on $\mathscr{M} \bar{\otimes} \mathscr{L}\left(l^{2}(G)\right)$, where $\rho$ is given by the right regular representation $\rho(\cdot)$ of $G$ on $l^{2}(G)$. By [16, Lemma 4.7], $\alpha$ and $\alpha^{\prime}$ are cocycle conjugate.

Clearly, $\phi$ is dominant and invariant under $\alpha^{\prime}$. Further, by [4, p. 497], there exists a one parameter unitary group $\{v(s): s \in \mathbf{R}\}$ in $\mathscr{M}$ with $\sigma_{t}^{\psi}(v(s))=e^{-i s t} v(s), s, t \in \mathbf{R}$. Since $\left\{\alpha_{g}: g \in \mathbf{R}\right\}$ and $\left\{\sigma_{t}^{\psi}: t \in \mathbf{R}\right\}$ commute, we have

$$
w(g, s)=\alpha_{g}(v(s)) v(s)^{*} \in \mathscr{M}_{\psi}, \quad g \in G, s \in \mathbf{R} .
$$


We then have, with $\theta_{s}=\operatorname{Ad}(v(s))$,

$$
\begin{aligned}
w(g, s+t) & =w(g, s) \theta_{s}(w(g, t)), & & g \in G, s, t \in \mathbf{R} ; \\
w(g h, s) & =\alpha_{g}(w(h, s)) w(g, s), & & h \in H .
\end{aligned}
$$

For each $t \in \mathbf{R}$, the map: $g \in G \rightarrow w(g, t)^{*}$ is in $Z_{\alpha}^{1}\left(G, \mathscr{U}\left(\mathscr{M}_{\psi}\right)\right)$. We set

$$
(w(t) \xi)(g)=w\left(g^{-1}, t\right)^{*} \xi(g), \quad \xi \in \mathscr{H} \otimes l^{2}(G) .
$$

It then follows that

$$
\alpha_{g}^{\prime}(w(t)) w(t)^{*}=w(g, t) \otimes 1,
$$

and that

$$
w(s)^{*}\left(\theta_{s} \otimes \mathrm{id}\right)\left(w(t)^{*}\right)=w(s+t)^{*} .
$$

Hence the map $s \in \mathbf{R} \rightarrow w(s)^{*}$ is in $Z_{(\theta \otimes l)}^{1}\left(\mathbf{R}, \mathscr{U}\left(\mathscr{M} \bar{\otimes} \mathscr{L}\left(l^{2}(G)\right)\right)_{\phi}\right)$. But $\theta \otimes l$ is stable on $\mathscr{M}_{\psi} \bar{\otimes} \mathscr{L}\left(l^{2}(G)\right)$ by [4, p. 497 and 544], so there is a unitary $w \in \mathscr{M}_{\psi} \bar{\otimes} \mathscr{L}\left(l^{2}(G)\right)$ with

$$
w(t)^{*}=w\left(\theta_{t} \otimes \mathrm{id}\right)\left(w^{*}\right), \quad t \in \mathbf{R} .
$$

It then follows that

$$
\begin{aligned}
w(t)^{*}(v(t) \otimes 1) & =w(v(t) \otimes 1) w^{*}, \quad t \in \mathbf{R} ; \\
\alpha_{g}^{\prime}\left(w(t)^{*}(v(t) \otimes 1)\right) & =w(t)^{*}\left(w(t, g)^{*} \otimes 1\right)\left(\alpha_{g}(v(t)) \otimes 1\right) \\
& =w(t)^{*}\left(w(t, g)^{*} \otimes 1\right)(w(t, g) v(t) \otimes 1) \\
& =w(t)^{*}(v(t) \otimes 1) ; \\
\sigma_{t}^{\phi}\left(w(s)^{*}(v(s) \otimes 1)\right) & =e^{-i s t} w(s)^{*}(v(s) \otimes 1) .
\end{aligned}
$$

Therefore, with $u(s)=w(v(s) \otimes 1) w^{*}$, we obtain the desired one parameter unitary group $\{u(s)\}$.

Replacing the action $\alpha$ by $\alpha^{\prime}$, we assume that $\alpha$ satisfies the conclusion (i), (ii) and (iii) of Lemma 5.11, and fixes the one parameter unitary group $\{u(s)\}$ of (iii).

LEMmA 5.12. In the above context, let $\alpha^{\prime}: H=G \times \mathbf{R} \rightarrow \operatorname{Aut}\left(\mathscr{M}_{\phi}\right)$ be the action defined by:

$$
\alpha_{(g, t)}^{\prime}=\alpha_{g} \circ \operatorname{Ad}(u(t))=\alpha_{g} \circ \theta_{t}, \quad(g, t) \in H,
$$

and choose a map:

$$
n \in N(\alpha) \rightarrow v_{\phi}(n)=(v(n), c(n)) \in \tilde{\mathscr{U}}_{\phi}(\mathscr{M})
$$

such that

$$
\alpha_{n}=\operatorname{Ad}_{\phi} v_{\phi}(n)=\operatorname{Ad}(v(n)) \circ \bar{\sigma}_{c(n)}^{\phi}, \quad n \in N(\alpha) .
$$


We then conclude the following.

(i) $\alpha_{(g, t)}^{\prime}$ is inner if and only if $(g, t) \in N(\alpha) \times\{0\}$;

(ii) For $n \in N(\alpha), v(n) \in \mathscr{M}_{\phi}$ and

$$
\alpha_{n}^{\prime}=\operatorname{Ad} v(n)
$$

(iii) The characteristic invariants of the restriction of $\alpha^{\prime}$ to $G \times\{0\} \subset$ $H$ coincide with the characteristic invariants of $\alpha$;

(iv) $\alpha_{t}^{\prime}(v(n))=c(n, t) v(n)$, $n \in N(\alpha), t \in \mathbf{R}$,

where $c(n, t)$ is the value of $c(n) \in Z^{1}(\mathscr{F}(\mathscr{M}))$ at $t$.

Proof. Let $\operatorname{Tr}$ be the faithful semi-finite normal trace on $\mathscr{M}_{\phi}$ such that the weight $\phi$ is dual to $\operatorname{Tr}$, so that $\operatorname{Tr} \circ \theta_{s}=e^{-s} \operatorname{Tr}$. Since $\operatorname{Tr}$ is the unique trace on $\mathscr{M}_{\phi}$ such that $\phi=\operatorname{Tr} \circ E_{\phi}$, where $E_{\phi}$ is the $\mathscr{M}_{\phi}$-valued weight:

$$
E_{\phi}(x)=\int_{\mathbf{R}} \sigma_{t}^{\phi}(x) d t, \quad x \in \mathscr{M}_{+},
$$

the $\alpha$-invariance of $\phi$ implies that $\operatorname{Tr} \circ \alpha_{g}^{\prime}=\operatorname{Tr}, g \in G$. Thus if $\alpha_{(g, t)}^{\prime}$ is inner, $t=0$. Also, if $\alpha_{g}=\operatorname{Ad} v(g)$ on $\mathscr{M}_{\phi}$, then $\operatorname{Ad}\left(v(g)^{*}\right) \circ \alpha_{g}$ is trivial on $\mathscr{M}_{\phi}$, so that $\alpha_{g}=\operatorname{Ad}(v(g)) \circ \bar{\sigma}_{c(g)}^{\phi}$ for some $c(g) \in Z^{1}(\mathscr{F}(\mathscr{M}))$ by [4, p. 557]. Since $\phi \circ \alpha_{g}=\phi, v(g)$ must be in $\mathscr{M}_{\phi}$, so that $g$ belongs to $N(\alpha)$. Thus (i) and (ii) are proven.

The assertion (iii) is obvious.

(iv) Since $\alpha_{n}(u(t))=u(t)$, we have

$$
u(t)=v(n) \bar{\sigma}_{c(n)}^{\phi}(u(t)) v(n)^{*}=v(n) c(n, t) u(t) v(n)^{*},
$$

which implies (5.25).

We are finally ready to complete the proof of our main theorem.

Proof of Theorem 5.9. Let $\alpha$ and $\beta$ be actions of $G$ on $\mathscr{M}$ as in the theorem. By Lemma 5.10, 5.11 and 5.12, we may change $\alpha$ and $\beta$ within their cocycle conjugance classes and assume

(i) $\alpha$ and $\beta$ both admit invariant dominant weights $\phi$ and $\psi$ respectively;

(ii) there are one parameter unitary groups $\{u(s)\}$ and $\{v(s)\}$ in $\mathscr{M}$ such that

$$
\begin{aligned}
\sigma_{t}^{\phi}(u(s))=e^{i s t} u(s), & \sigma_{t}^{\psi}(v(s))=e^{i s t} v(s) \\
\alpha_{g}(u(s))=u(s), & \beta_{g}(v(s))=v(s)
\end{aligned}
$$

for $g \in G$ and $s, t \in \mathbf{R}$. 
Let $\alpha^{\prime}$ and $\beta^{\prime}$ be the actions of $H=G \times \mathbf{R}$ on $\mathscr{M}_{\phi}$ and $\mathscr{M}_{\psi}$ respectively as described in Lemma 5.12. Observe that $\alpha^{\prime}$ and $\beta^{\prime}$ both satisfy Assumption 4.1. By the hypothesis of Theorem 3.5 for $\alpha$ and $\beta$, there is an isomorphism $\theta$ of $\mathscr{C}_{\phi}$ onto $\mathscr{C}_{\psi}$ (which may be extended to an isomorphism of $\mathscr{M}_{\phi}$ onto $\mathscr{M}_{\psi}$ ), which intertwines the actions $\alpha^{\prime}$ on $\mathscr{C}_{\phi}$ and $\beta^{\prime}$ on $\mathscr{C}_{\psi}$, and which carries the characteristic invariants for $\alpha^{\prime}$ to those for $\beta^{\prime}$.

By Theorem 4.2, the actions $\alpha^{\prime}$ and $\beta^{\prime}$ of $H=G \times \mathbf{R}$ on $\mathscr{M}_{\phi}$ and $\mathscr{M}_{\psi}$ are cocycle conjugate. Now form the partial crossed products $\mathscr{M}_{\phi} \ltimes \mathbf{R}$ and $\mathscr{M}_{\psi} \ltimes \mathbf{R}$ and the actions $\bar{\alpha}^{\prime}$ and $\bar{\beta}^{\prime}$ (of $\S 1$ ) of $G$ on these algebras. By Proposition 1.1, these actions are cocycle conjugate. However, $\bar{\alpha}^{\prime}$ is nothing but $\alpha$, and the same is true for $\bar{\beta}^{\prime}$ and $\beta$. Therefore, $\alpha$ and $\beta$ are cocycle conjugate.

Remark 5.13. Our conclusions, up to Lemma 5.12, are all valid for any factor $\mathscr{M}$ of type III and for any action of any separable locally compact group $G$.

Finally, we turn to the problem of realization of a given set of invariants by a suitable action. This is not completely straightforward, as we shall see. One form of solution is, however, easy to give.

THEOREM 5.14. Let $\beta: G \rightarrow \operatorname{Aut}(\mathscr{F}(\mathscr{M}))$ be given, and let

$$
(\chi, \nu) \in \Lambda_{\beta}(G, N, \mathscr{U}(\mathscr{F}(\mathscr{M}))) \times \operatorname{Hom}\left(N, H^{1}(\mathscr{F}(\mathscr{M}))\right),
$$

where $N$ is a normal subgroup acting trivially on $\mathscr{F}(\mathscr{M})$ via $\beta$. Then there is an action $\alpha$ of $G$ on $\mathscr{M}$ such that

$$
\left(\bmod \alpha, \chi_{\alpha}, \nu_{\alpha}\right)=(\beta, \chi, \nu)
$$

if and only if

(i) $\chi \in \Lambda_{\beta}(G, N, \mathscr{U}(\mathscr{F}(\mathscr{M})))^{\mathbf{R}}$;

(ii) $\delta(\chi, \nu)=1$,

where $\delta$ is the homomorphism appearing in the exact sequence (2.4).

Proof. We have seen in Lemma 5.12 that if

$$
(\beta, \chi, \nu)=\left(\bmod \alpha, \chi_{\alpha}, \nu_{\alpha}\right)
$$

for some action $\alpha$ of $G$ on $\mathscr{M}$, then $(\chi, \nu)$ is the restriction of the 
characteristic invariant $\chi_{\alpha}$, for the action $\alpha^{\prime}$ of $H=G \times \mathbf{R}$ on $\mathscr{M}_{\phi}$ given in Lemma 5.12, so that $\delta(\chi, \nu)=1$ by Theorem 2.2.

Conversely, suppose that $(\chi, \nu)$ satisfies (i) and (ii). Since $\beta$ commutes with the flow of weights, it extends to an action $\alpha$ of $H=$ $G \times \mathbf{R}$ on $\mathscr{F}(\mathscr{M})=\mathscr{A}$. By Theorem 2.2 (ii), there exists $\chi^{\prime} \in$ $\Lambda_{\alpha}(G \times \mathbf{R}, N, \mathscr{U}(\mathscr{A}))$ such that the restriction of $\chi^{\prime}$ to $G$ and $\mathbf{R}$ gives $(\chi, \nu)$. By Theorem 4.4, there exists an action $\alpha^{\prime}$ of $G \times \mathbf{R}$ on $\mathscr{A} \bar{\otimes} \mathscr{R}_{0,1}$ satisfying Assumption 4.1 such that (i) $\alpha^{\prime}$ extends $\alpha$, and (ii) $\chi_{\alpha^{\prime}}=\chi^{\prime}$. Now, let $\mathscr{M}=\left(\mathscr{A} \bar{\otimes}_{\mathscr{R}_{0,1}}\right) \ltimes_{\alpha} \mathbf{R}$ be the partial crossed product, and take $\alpha$ as the canonical action $\alpha^{\prime}$ of $G$ on $\mathscr{M}$. We then have $\left(\chi_{\alpha}, \nu_{\alpha}\right)=(\chi, \nu)$.

\section{REFERENCES}

[1] A. Connes, Periodic automorphisms of the hyperfinite factor of type $\mathrm{II}_{1}$, Acta Sci. Math., 39 (1977), 39-66.

[2] - Outer conjugacy classes of automorphisms of factors, Ann. Sci. Ec. Norm. Sup. 4 series, t. 8 (1975), 383-420.

[3] On the classification of von Neumann algebras and their automorphisms, Symposia Math., XX (1976), 435-578.

[4] A. Connes and M. Takesaki, The flow of weights on factors of type III, Tôhoku Math. J., 29 (1977), 473-575.

[5] J. Feldman, P. Hahn and C. C. Moore, Orbit structure and countable sections for actions of continuous groups, J. Funct. Anal., 28, No. 3 (1978), 186-230.

[6] A. Ionescu-Tulcea, On the existence of a lifting commuting with left translations of an arbitrary locally compact group, Proc. Vth Berkeley Symp. on Math. Stat. and Probability, Univ. of California Press (1967), 63-97.

[7] V. F. R. Jones, Actions of finite groups on the hyperfinite $\mathrm{II}_{1}$ factor, Memoirs Amer. Math. Soc. No. 237 (1980).

[8] V. F. R. Jones and M. Takesaki, Actions of compact abelian groups on semifinite injective factors, Acta Math., 153 (1984), 213-258.

[9] C. C. Moore, Group extensions and cohomology for locally compact groups III, Trans. Amer. Math. Soc., 221 (1976), 1-36.

[10] Y. Nakagami and M. Takesaki, Duality for Crossed Products of von Neumann Algebras, Springer Lecture Notes in Mathematics, No. 731 (1979).

[11] A. Ocneanu, Actions of Discrete Amenable Groups on von Neumann Algebras, Springer Lecture Notes in Mathematics, 1138 (1985).

[12] A. Ramsay, Topologies on Measured Groupoids, J. Funct. Anal., 47, No. 3 (1982), 314-343.

[13] S. Stratila, Modular Theory in Operator Algebras, Editura Academiei and Abacus Press, (1981).

[14] C. E. Sutherland, Cohomology and Extensions of von Neumann Algebras I, R.I.M.S. Kyoto University, 16, No. 1 (1980), 105-133.

[15] C. E. Sutherland and M. Takesaki, Actions of Discrete Amenable Groups and Groupoids on von Neumann Algebras, R.I.M.S. Kyoto University, 21, No. 6 (1985), 1087-1120. 
[16] M. Takesaki, Duality for crossed products and the structure of von Neumann algebras of type III, Acta Math., 131 (1973), 249-310.

[17] _ , Theory of Operator Algebras I, Springer-Verlag, New York (1979).

Received September 24, 1987. This research was supported in part by NSF Grant No. DMS 86-03223, and by the A.R.G.S., Australia.

UNIVERSITY OF New SOUTH WaLeS

Kensington NSW 2033, Australia

AND

University of California, Los ANGeles

LOS ANGELES, CA 90024-1555

USA 


\section{PACIFIC JOURNAL OF MATHEMATICS EDITORS}

\author{
V. S. VARADARAJAN \\ (Managing Editor) \\ University of California \\ Los Angeles, CA 90024 \\ Herbert Clemens \\ University of Utah \\ Salt Lake City, UT 84112 \\ THOMAS ENRIGHT \\ University of California, San Diego \\ La Jolla, CA 92093
}

R. FINN

Stanford University

Stanford, CA 94305

HeRmann FlaschKa

University of Arizona

Tucson, AZ 85721

VAUGHAN F. R. Jones

University of California

Berkeley, CA 94720

STEVEN KeRCKHOFF

Stanford University

Stanford, CA 94305
ROBION KIRBY

University of California

Berkeley, CA 94720

C. C. MOORE

University of California

Berkeley, CA 94720

HAROLD STARK

University of California, San Diego

La Jolla, CA 92093

\section{ASSOCIATE EDITORS}
R. ARENS
E. F. BECKENBACH
B. H. NeUMANN
F. WolF
K. YoshidA (1906-1982)

\section{SUPPORTING INSTITUTIONS}

UNIVERSITY OF ARIZONA

UNIVERSITY OF BRITISH COLUMBIA

CALIFORNIA INSTITUTE OF TECHNOLOGY

UNIVERSITY OF CALIFORNIA

MONTANA STATE UNIVERSITY

UNIVERSITY OF NEVADA, RENO

NEW MEXICO STATE UNIVERSITY

OREGON STATE UNIVERSITY

\author{
UNIVERSITY OF OREGON \\ UNIVERSITY OF SOUTHERN CALIFORNIA \\ STANFORD UNIVERSITY \\ UNIVERSITY OF HAWAII \\ UNIVERSITY OF TOKYO \\ UNIVERSITY OF UTAH \\ WASHINGTON STATE UNIVERSITY \\ UNIVERSITY OF WASHINGTON
}

The Supporting Institutions listed above contribute to the cost of publication of this Journal, but they are not owners or publishers and have no responsibility for its content or policies.

Mathematical papers intended for publication in the Pacific Journal of Mathematics should be in typed form or offset-reproduced (not dittoed), double spaced with large margins. Please do not use built up fractions in the text of the manuscript. However, you may use them in the displayed equations. Underline Greek letters in red, German in green, and script in blue. The first paragraph must be capable of being used separately as a synopsis of the entire paper. In particular it should contain no bibliographic references. Please propose a heading for the odd numbered pages of less than 35 characters. Manuscripts, in triplicate, may be sent to any one of the editors. Please classify according to the scheme of Math. Reviews, Index to Vol. 39. Supply name and address of author to whom proofs should be sent. All other communications should be addressed to the managing editor, or Elaine Barth, University of California, Los Angeles, California 90024.

There are page-charges associated with articles appearing in the Pacific Journal of Mathematics. These charges are expected to be paid by the author's University, Government Agency or Company. If the author or authors do not have access to such Institutional support these charges are waived. Single authors will receive 50 free reprints; joint authors will receive a total of 100 free reprints. Additional copies may be obtained at cost in multiples of 50 .

The Pacific Journal of Mathematics is issued monthly as of January 1966. Regular subscription rate: $\$ 190.00$ a year (5 Vols., 10 issues). Special rate: $\$ 95.00$ a year to individual members of supporting institutions.

Subscriptions, orders for numbers issued in the last three calendar years, and changes of address should be sent to Pacific Journal of Mathematics, P.O. Box 969, Carmel Valley, CA 93924, U.S.A. Old back numbers obtainable from Kraus Periodicals Co., Route 100, Millwood, NY 10546.

The Pacific Journal of Mathematics at P.O. Box 969, Carmel Valley, CA 93924 (ISSN 0030-8730) publishes 5 volumes per year. Application to mail at Second-class postage rates is pending at Carmel Valley, California, and additional mailing offices. Postmaster: send address changes to Pacific Journal of Mathematics, P.O. Box 969, Carmel Valley, CA 93924.

\section{PUBLISHED BY PACIFIC JOURNAL OF MATHEMATICS, A NON-PROFIT CORPORATION}




\section{Pacific Journal of Mathematics}

Vol. 137, No. $2 \quad$ February, 1989

Alain Connes and E. J. Woods, Hyperfinite von Neumann algebras and Poisson boundaries of time dependent random walks ............ 225

R. Coquereaux and D. Kastler, Remarks on the differential envelopes of

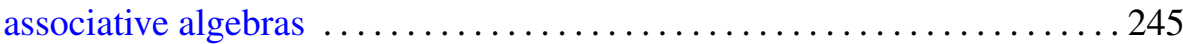

Uffe Haagerup, The injective factors of type $\mathrm{III}_{\lambda}, 0<\lambda<1 \ldots \ldots \ldots 265$

Vaughan Jones, On knot invariants related to some statistical mechanical

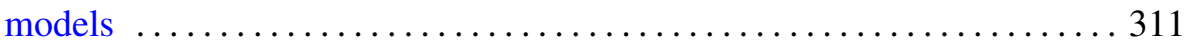

Paul Scott Muhly, Kichi-Suke Saito and Baruch Solel, Coordinates for triangular operator algebras. II ......................... 335

Klaus Schmidt, Mixing automorphisms of compact groups and a theorem by Kurt Mahler ........................................ 371

Irving E. Segal, Algebraic characterization of the vacuum for quantized

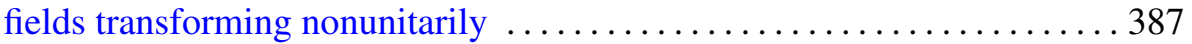

Colin Eric Sutherland and Masamichi Takesaki, Actions of discrete amenable groups on injective factors of type $\mathrm{III}_{\lambda}, \lambda \neq 1 \ldots \ldots \ldots \ldots 405$ 